\title{
EXTREME ULTRAVIOLET AND SOFT X-RAY DIAGNOSTICS \\ OF HIGH-TEMPERATURE PLASMAS
}

Annual Progress Report

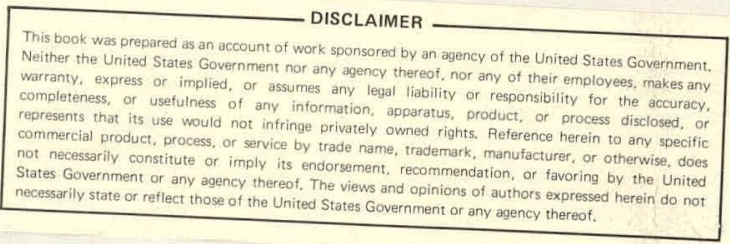

Prepared By

H. Warren Moos and L. Armstrong, Jr.

The Johns Hopkins University

Baltimore, Maryland

DISTABQUTION OF THIS BOCUMEXT IS UNLAMTED

June 1, 1979 - September 30, 1980

PREFARED FOR THE U. S. DFPARTMENT OF ENERGY

UNDER CONTRACT DE-AS02-76ET53006 


\section{DISCLAIMER}

This report was prepared as an account of work sponsored by an agency of the United States Government. Neither the United States Government nor any agency Thereof, nor any of their employees, makes any warranty, express or implied, or assumes any legal liability or responsibility for the accuracy, completeness, or usefulness of any information, apparatus, product, or process disclosed, or represents that its use would not infringe privately owned rights. Reference herein to any specific commercial product, process, or service by trade name, trademark, manufacturer, or otherwise does not necessarily constitute or imply its endorsement, recommendation, or favoring by the United States Government or any agency thereof. The views and opinions of authors expressed herein do not necessarily state or reflect those of the United States Government or any agency thereof. 


\section{DISCLAIMER}

Portions of this document may be illegible in electronic image products. Images are produced from the best available original document. 
RESEARCH STAFF :

Professor H. Warren Moos, Co-Research Contract Director

Professor L. Armstrong, Jr., Co-Research Contract Director

Professor Wm. G. Fastie

William L. Hodge, Jr., Associate Research Scientist

Raymond J. Dukart

Postdoctoral Fellow

Michael Finkenthal

Postdoctoral Fellow

Dinh Ton-That

Postdoctoral Fellow

Mina V.-Samii

Postdoctoral Fellow

Steven Allen

Research Assistant

Ronald Bell

Research Assistant

James Castracane

Research Assistant

William Fielder

Research Assistant

Richard Holman

Research Assistant

Oliver T. Strand

Research Assistant

Brent Stratton

Research Assistant 
TABLE OF CONTENTS

RESEARCH STAFF

Page

ABSTRACT

iv

I. INTRODUCTION

1

II. THEORY

A. Transition Energies and Oscillator Strengths Along Isoelectronic Sequences

B. Photoionization

C. Electron-Atom Scattering

III. DIAGNOSTIC STUDIES

$\begin{array}{ll}\text { A. Alcator A } & 10\end{array}$

B. Alcator C 36

C. The Tandem Mirror Experiment $\quad 40$

IV. LABORATORY STUDIES

A. Calibration of Grazing Incidence Instrument 51 with the SURF Facility

B. Grating Efficiencies $\quad 54$

$\begin{array}{ll}\text { C. Tokamak Line Study } & 57\end{array}$

V. INSTRUMENTATION

A. Time-Resolving EUV Spectrograph 62

B. Two Meter Grazing Incidence Double Monochromator 73

C. Time-Resolving Grazluy Incidence Spectrograph 74

$\begin{array}{ll}\text { VI. PUBLICATIONS AND ABSTRACTS } & 76\end{array}$ 
ABSTRACT

The work performed from mid-January 1979 through 1980 is described. EUV diagnostic studies have been performed at the Alcator A and C Tokamaks, and on TMX. A toroidal asymmetry has been found in the low density phase of Alcator A. The confinement time of impurities has been measured on Alcator A. Impurity concentrations in the center cell of TMX have been studied using EUV spectroscopic techniques. A time resolving spectrograph with 1024 detector elements is essentially complete.

This report was prepared as an account of Government-sponsored work. Neither the United States, nor the Department of Energy nor any person acting on behalf of the Commission,

A. Makes any warranty or representation, expressed or implied, with respect to the accuracy, completeness, or usefulness of the information contained in this report, or that the use of the information, apparatus, method, or process disclosed in this report may not infringe privately owned rights; or

B. Assumes any liabilities with respect to the use of, or for damages resulting from the use of, any information, apparatus, method, or process disclosed in this report.

As used in the ahove, "person acting on behalf of the Commission" includes any employee or contractor of the Department of Energy or employee of such contractor, to the extent that such employee or contractor of the Department of Energy or employee of such contractor prepares, disseminates, or provides access to, any information pursuant to his employment or contract with the Department of Energy or his employment with such contractor. 


\section{INTRODUCTION}

This report describes the work performed by this laboratory for the Magnetic Fusion Energy program of the Department of Energy from mid January 1979 through April, 1980.

The program divides naturally into several areas of effort. These areas of effort are covered in separate sections. Theoretical atomic structural calculation in support of MFE diagnostics are described in section II. Section III covers the diagnostic studies of plasma devices. Supporting laboratory studies are described in Section IV. Finally in Section $V$ we discuss specific instrumentation systems which are under development for diagnostic studies. The names of personnel associated with each area are listed at the beginning of each section.

The diagnostic work on plasma devices continues to be a major part of our work. The spatial imaging detector system has been used to measure toroidal transport and impurity confinement in the Alcator A tokamak. The extent of radiative losses due to molybdenum have been measured on the Alcator A and C tokamaks. The impurity diagnostic experiments have continued and additional instrumentation for transpor't study has been installed. The time resolving spectrograph with 1024 detector elements has been completed and is undergoing tests prior to installation on Alcator A. $\cdots$ Development of. a similar system for the grazing incidence region has begun.

The major emphasis of the theoretical program continues to be on the calculation of transition energies and oscillator strengths along 1soelectronic sequences. Several sequences have been considered during the present contract period. We have also carried out calculations in photoionization and electron impact excitation of atoms. 


\section{THEORY}

(L. Armstrong, W. Fielder, D. Ton-That, M. V-Samii)

\section{Introduction}

During the contract period, we have continued to work in the general areas of transition energies and probabilities along isoelectronic sequences; electron-atom scattering; and photoionization. Several projects were completed in these areas, and several are still in progress. Each of these areas will be discussed separately below. We have also begun to move our programs from the computers at Hopkins to the MFE computer at Livermore. At present, the programs needed in the first area mentioned above are running at Livermore. This will also be discussed further in the appropriate section below.

\section{II.A. Transition Energies and Oscillator Strengths Along Isoelectronic}

\section{Sequences}

This area remains the dominant part of this theoretical program. Multiconfiguration. Dirac Hartree Fock (MDHF) calculations are made along isoelectronic sequences in order to calculate transition energies and oscillator strengths of large numbers of 1ons. During the present contract period, we reported on E1 and M2 transitions in the Ne sequence ${ }^{1}$, E1 transitions in the $B$ sequence ${ }^{2}$, and $M$ transitions ${ }^{2}$ for all the flrot row atoms (see examples in Tables II.A-1 and II.A-2)

The results obtalned thus far for the $B$ sequence have been particularly interesting because of their poor quality. The general approach used in previous calculations has been to include in the MDHF all configurations composed of electrons belonging to the ground state complex, 1.e. all those having the same principal quantum numbers as the electrons in the 
partially filled shells of the ground state. In previous calculations, this has led to very good agreement between the length and velocity forms of the E1 transition operator. Such agreement is, of course, a necessary but not sufficient indication of the accuracy of the wavefunctions. However, in the B sequence, quite large discrepencies between length and velocity forms (of the order of $50 \%$ ) remain for the stronger transitions even for fairly highly ionized atoms (e.g. B-like Fe, see Table II.A-1). Such discrepencies have also been privately reported by others working on the same problem.

We are continuing to work on the B sequence in an effort to improve the present results. Unfortunately, the MDHF program we use (Desclaux ${ }^{3}$ ) often has difficulty in converging to excited virtual orbitals of the types needed to improve the present results. We are investigating a number of alternate approaches at this time and have obtained some small improvement in the values of the oscillator strengths.

As noted above, we have shifted a number of our computer programs from the local computer to the MFE computer in Livermore. In order to communicate with the Livermore computer, we purchased a Tektronix 4025 terminal with a DEC-writer to provide hard copy. We fuund the probleme associated with the shift to be much larger and more time consuming than expected, primarily because of the nonstandard and quite inefficient compiler (CHATR) which is used on the CDC 7600 . All programs seem to be running now, although we did lose a great deal of time in making the shift.

\section{II.B. Photoionization}

We have finished our calculation of photoionization cross section and angular distribution in chlorine. This problem was investigated using 
a mixture of techniques: multiconfiguration Hartree Fock was used to evaluate ground state correlations; multiconfiguration $\mathrm{V}^{(\mathrm{N}-1) \mathrm{LS}}$ was used to evaluate intrachannel final state interactions; and $\mathrm{K}$-matrix techniques were used to evaluate interchannel final state interactions. A preliminary report of our results was given at the Nobel Symposium on Many-Body Theory in Lund, Sweden ${ }^{5}$.

Chlorine is very interesting from a theoretical point of view because it is one of the flrst open shell atoms for which photoionization calculations have been made. A total of four other groups have now reported their calculations on chlorine, and agreement betwepn the different rcoults is not particularly good ${ }^{6}$. This is in strong contrast to the situation which occurs for the closed shell Ar atom, with one electron more than $\mathrm{Cl}$, where all methods give almost identical results. The differences in results serve to emphasize the tremendous difficulty of making accurate calculations in open shell atoms.

One interesting result of our calculations was the importance of one-electron excitations in the description of the ground and ionic states. One of the well known techniques for carrying out approximate many body calculations, the random phase approximation (RPA), neglects such excitations completely. We intend to make a study in the near future of oscillator strengths in the $\mathrm{Cl}$ sequence in order to situdy the importance of such excitations on bound-bound transitions.

\section{II.C. Electron-Atom Scattering}

We began our investigation ${ }^{7}$ of electron-atom scattering with a study of the sensitivity of Born excitation cross sections for the rare gases to the quality of target state wavefunctions. We used Hartree-Slater, Hartree-Fock, and multiconfiguration Hartree-Fock approximations for the 
target wavefunctions. Our results showed a great variation (factors of 2 - 3 ) in excitation cross sections as we improved the quality of the target wavefunctions. These results were reported at the Second Topical Conference on Atomic Processes in High Temperature Plasmas last year.

We have begun to study the possibility of using K-matrix techniques for scattering calculations. Solution of the K-matrix equations is, in principal, equivalent to solution of close coupling equations. The possible advantage of $\mathrm{K}$-matrix over close coupling is that the former involves solving algebraic equations, whereas the latter involves solving differential equations.

Our original hope was that we could use more-or-less directly the techniques developed for the photoionization calculations discussed above. However, it soon became clear that the basis sets required in this approach are too large to be useful in many-channel scattering problems. However, recent work by McKoy ${ }^{8}$ and Macek $^{9}$ has raised the possibility that smaller basis sets will suffice if the Schwinger form of the $K$ matrix equation is used. We are currently investigating this possibility. 
Table II.A-1

Osclilator strengths and Transition energies for E1 transition In the Boron isoelectrontc sequence:

$$
\left[2 s 2 p_{\frac{1}{2}}^{2}+2 s 2 p_{\frac{1}{2}} 2 p_{3 / 2}+2 s 2 p_{3 / 2}^{2}\right] ; J=\frac{1}{2}\left({ }^{2} s_{\frac{3}{2}}\right)+\left[2 s^{2} 2 p_{\frac{1}{2}}+2 p_{\frac{1}{2}} 2 p_{3 / 2}^{2}\right] ; J=\frac{1}{2}\left({ }^{2} p_{\frac{1}{2}}^{0}\right)
$$

\begin{tabular}{|c|c|c|c|c|}
\hline$z$ & Ion & $\Delta E\left(a_{\cdot} u_{\text {. }}\right)$ & $\mathbf{f}_{\ell}$ & $f_{v}$ \\
\hline 5 & B & 0.3159 & 0.1540 & 0.0205 \\
\hline 6 & $c^{+1}$ & 0.4706 & 0.1228 & 0.0181 \\
\hline 7 & $\mathrm{~N}^{+2}$ & 0.6225 & 0.0991 & 0.0161 \\
\hline 8 & $0^{+3}$ & 0.7733 & 0.0832 & 0.0147 \\
\hline 10 & $\mathrm{Ne}^{+5}$ & 1.0755 & 0.0652 & 0.0133 \\
\hline 12 & $\mathrm{Mg}^{+7}$ & 1.3827 & 0.0578 & 0.0139 \\
\hline 13 & $\mathrm{Al}^{+8}$ & 1.5396 & 0.0568 & 0.0150 \\
\hline 14 & $\mathrm{Si}^{+9}$ & 1.6994 & 0.0573 & 0.0167 \\
\hline I6 & $s^{+11}$ & 2.0293 & 0.0623 & 0.0223 \\
\hline 18 & $\mathrm{Ar}^{+1.3}$ & 2.3746 & 0.0713 & 0.0308 \\
\hline 22 & $\mathrm{TI}^{+17}$ & 3.1139 & 0.0835 & 0.0453 \\
\hline 23 & $v^{+18}$ & 3.3107 & 0.0836 & 0.0468 \\
\hline 26 & $\mathrm{Fe}^{+21}$ & 3.9438 & 0.0802 & 0.0482 \\
\hline 28 & $\mathrm{~N} 1^{+23}$ & 4.4132 & 0.0770 & 0.0480 \\
\hline 29 & $\mathrm{Cu}^{+24}$ & 4.6661 & 0.0754 & 0.0479 \\
\hline 36 & $\mathrm{Kr}^{+31}$ & 6.9248 & 0.0663 & 0.0482 \\
\hline 42 & $\mathrm{Mo}^{+37}$ & 9.8759 & 0.0625 & 0.0503 \\
\hline 54 & $x e^{+49}$ & 20.5515 & 0.0661 & 0.0603 \\
\hline 74 & $\mathrm{w}^{+69}$. & 65.6128 & 0.0951 & 0.0929 \\
\hline 82 & $\mathrm{~Pb}^{+77}$ & 100.8461 & 0.1128 & 0.1114 \\
\hline 92 & $u^{+87}$ & 168.6960 & 0.1395 & \\
\hline
\end{tabular}


Table II.A-2a

MI transition energies, rates and oscillator strengths for the Nitrogen isoelectronic sequence:

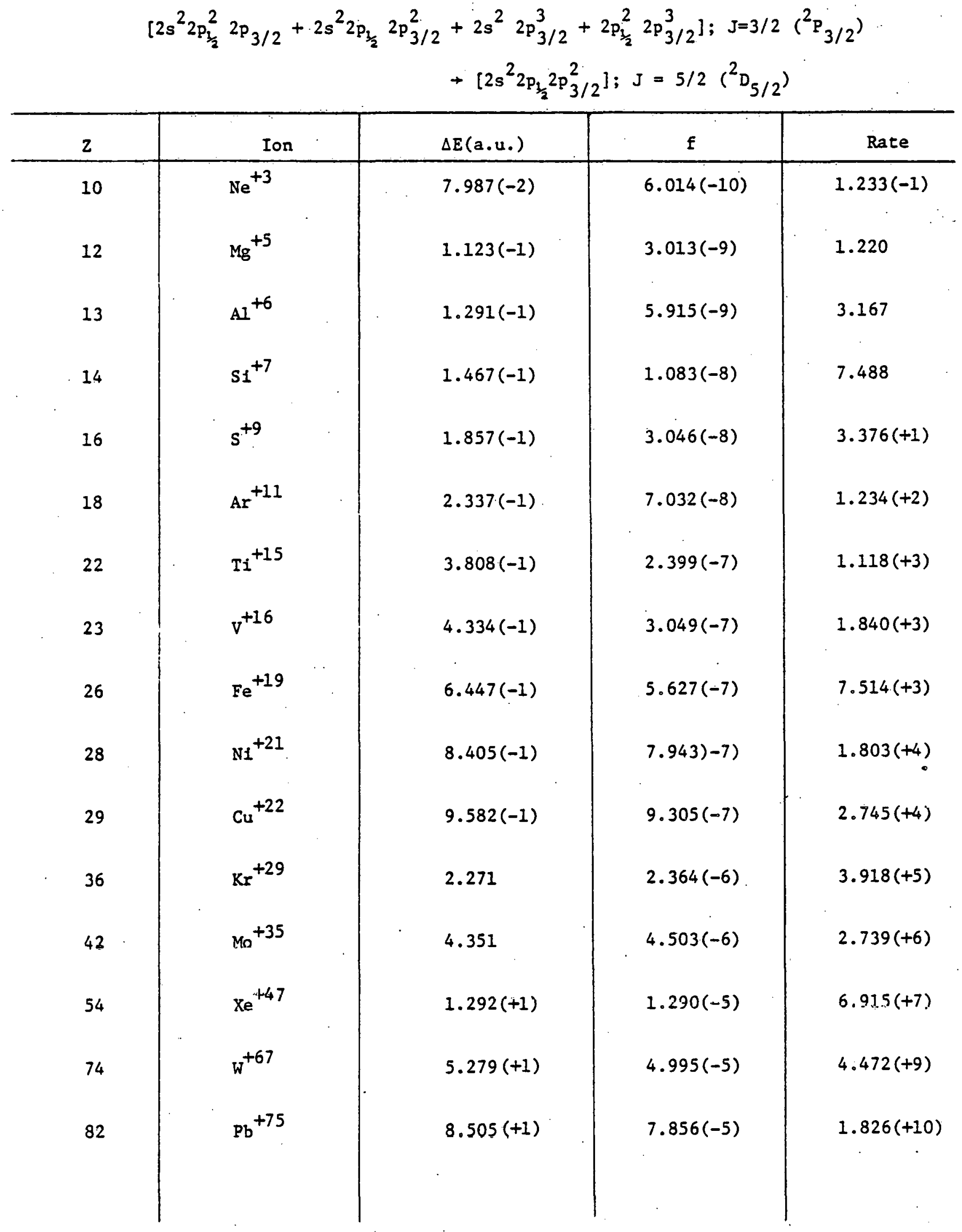


Mn transition energies, rates and oscillator strengths for the Nitrogen isoelectronic sequence:

$$
\begin{aligned}
& {\left[2 s^{2} 2 p_{\frac{1}{2}}^{2} 2 p_{3 / 2}+2 s^{2} 2 p_{\frac{3}{2}} 2 p_{3 / 2}^{2}+2 s^{2} 2 p_{3 / 2}^{3}+2 p_{\frac{1}{2}}^{2} 2 p_{3 / 2}^{3}\right] ; J=3 / 2\left({ }^{2} p_{3 / 2}\right)} \\
& +\left[2 s^{2} 2 p_{\frac{3}{2}}^{2} 2 p_{3 / 2}+2 s^{2} 2 p_{\frac{3}{2}} 2 p_{3 / 2}^{2}+2 s^{2} 2 p_{3 / 2}^{3}+2 p_{\frac{1}{2}}^{2} 2 p_{3 / 2}^{3}\right] ; J=3 / 2\left({ }^{2} \mathrm{D}_{3 / 2}\right)
\end{aligned}
$$

\begin{tabular}{|c|c|c|c|c|}
\hline $\mathbf{z}$ & Ion & $\Delta E\left(a_{0} u_{*}\right)$ & $\mathbf{f}$ & Rate \\
\hline 10 & $\mathrm{Ne}^{+3}$ & $7.996(-2)$ & $1.473(-9)$ & $3.026(-1)$ \\
\hline 12 & $\mathrm{Mg}^{+5}$ & $1.128(-1)$ & $7.879(-9)$ & 3.223 \\
\hline 13 & $\mathrm{Al}^{+6}$ & $1.303(-1)$ & $1.556(-8)$ & 8.494 \\
\hline 14 & $\mathrm{SI}^{+7}$ & $1.491(-1)$ & $2.858(-8)$ & $2.042(+1)$ \\
\hline 16 & $s^{+9}$ & $1.933(-1)$ & $8.097(-8)$ & $9.722(+1)$ \\
\hline 18 & $\mathrm{Ar}^{+11}$ & $2.527(-1)$ & $1.9 .90(-7)$ & $3.897(+2)$ \\
\hline 22 & $\mathrm{TI}^{+15}$ & $4.558(-1)$ & $7.034(-7)$ & $4.696(+3)$ \\
\hline 23 & $v^{+16}$ & $5.312(-1)$ & $9.247(-7)$ & $8.384(+3)$ \\
\hline 26 & $\mathrm{Fe}^{+19}$ & $8.308(-1)$ & $1.947(-6)$ & $4.317(+4)$ \\
\hline 28 & $\mathrm{Ni}^{+21}$ & 1.096 & $3.030(-6)$ & $1.171(+5)$ \\
\hline 39 & $\mathrm{Cu}^{+22}$ & 1.250 & $3.716(-6)$ & $1.866(+5)$ \\
\hline 36 & $\mathrm{Kr}^{+29}$ & 2.783 & $1.153(-5)$ & $2.868(+6)$ \\
\hline 42 & $\mathrm{Mo}^{+35}$ & 5.002 & $2.359(-5)$ & $1.896(+7)$ \\
\hline 54 & $x e^{+47}$ & $1.380(+1)$ & $7.243(-5)$ & $4.429(+8)$ \\
\hline 74 & $\mathrm{w}^{+67}$ & $5.401(+1)$ & $2.922(-4)$ & $2.739(+10)$ \\
\hline 82 & $\mathrm{~Pb}^{+75}$ & $8.641(+1)$ & $4.628(-4)$ & $1.110(+11)$ \\
\hline
\end{tabular}




\section{II.D References}

1. W. F. Fielder, D. L. Lin, and D. Ton-That, Phys. Rev. 19, 741 (1979).

2. M. V.-Samii, D. Ton-That, I. Armstrong, reported at the Fourth Johns Hopkins Workshop on Current Problems in Physics, March 1980, and submitted to the Atomic Data for Fusion Bulletin.

3. J. P. Desclaux, Comput. Phys. Commun. 9, 31 (1975).

4. J. R. Swanson and L. Armstrong, Phys. Rev. A 16, 1117 (1977).

5. L. Armstrong and W. R. Fielder, Physica Scriptica, 21, 457 (1980).

6. A. F. Starace and L. Armstrong, Phys. Rev. A 13 , 1850 (1976); N. A. Cherepkov and L. V. Chernysheva, Phys. Lett 60A, 103 (1977); E. P. Brown, S. L. Carter and H. P. Kel1y, Phys. Lett 66A, 290 (1978); M. Lamoureux and F. Combet Farnoux, Journal de Physique 40; 545 (1979).

7. D. Ton-That and L. Armstrong, reported at the Second Topical Conference on Atomic Processes in High Temperature Plasmas.

8. D. K. Watson, R. A. Luchese, V. Mckoy, and T. N. Rescigno, Phys. Rev. A 21, 738 (1980).

9. N. Maleki and J. Macek, to be published, Phys. Rev. A. 
III. DIAGNOSTIC STUDIES

(S. Allen, J. Castracane, W. Hodge, R. Holman, W. Moos, T. Strand, B. Stratton)

\section{Introduction}

This section describes the work on three plasma machines. In subsection A the Alcator A tokamak at M.I.T. is discussed; studies of radial transport, toroidal transport, identification of spectra by spatial profile, and radiative losses due to molybdenum are described. Section B covers light impurity concentrations and molybdenum radiative losses in the Alcator C tokamaks. Section C describes a series of EUV measurements on the TMX device at LLL.

\section{III.A. Alcator A}

III.A.1. $\therefore$ Radial Impuri'ty Transport

The study of impurity transport with naturally occurring impurities is very difficult; the source functions are ambiguous and recycling of the impurities may occur. Many previous experiments have been performed by artificially introducing gas impurities into the plasma; these are again plagued by recycling and the diffuse nature of the source. For this reason, a laser impurity injection experiment was started in January 1979. The laser injection technique hàs several advantages: 1) metal Impurities are introduced in a nearly delta-functinn manner; 2) the exact time of the injection is determined by the firing time of the pulsed laser; 3) by measuring the size of the spot on the slide, the exact number of injected impurity atoms can be determined; 4) the metal impurities recycle less. The experimental set-up is shown in Fig. III-1.

The initial experiments were carried out with molybdenum, both to test the injection apparatus and to find the effect of the limiter-1ike 


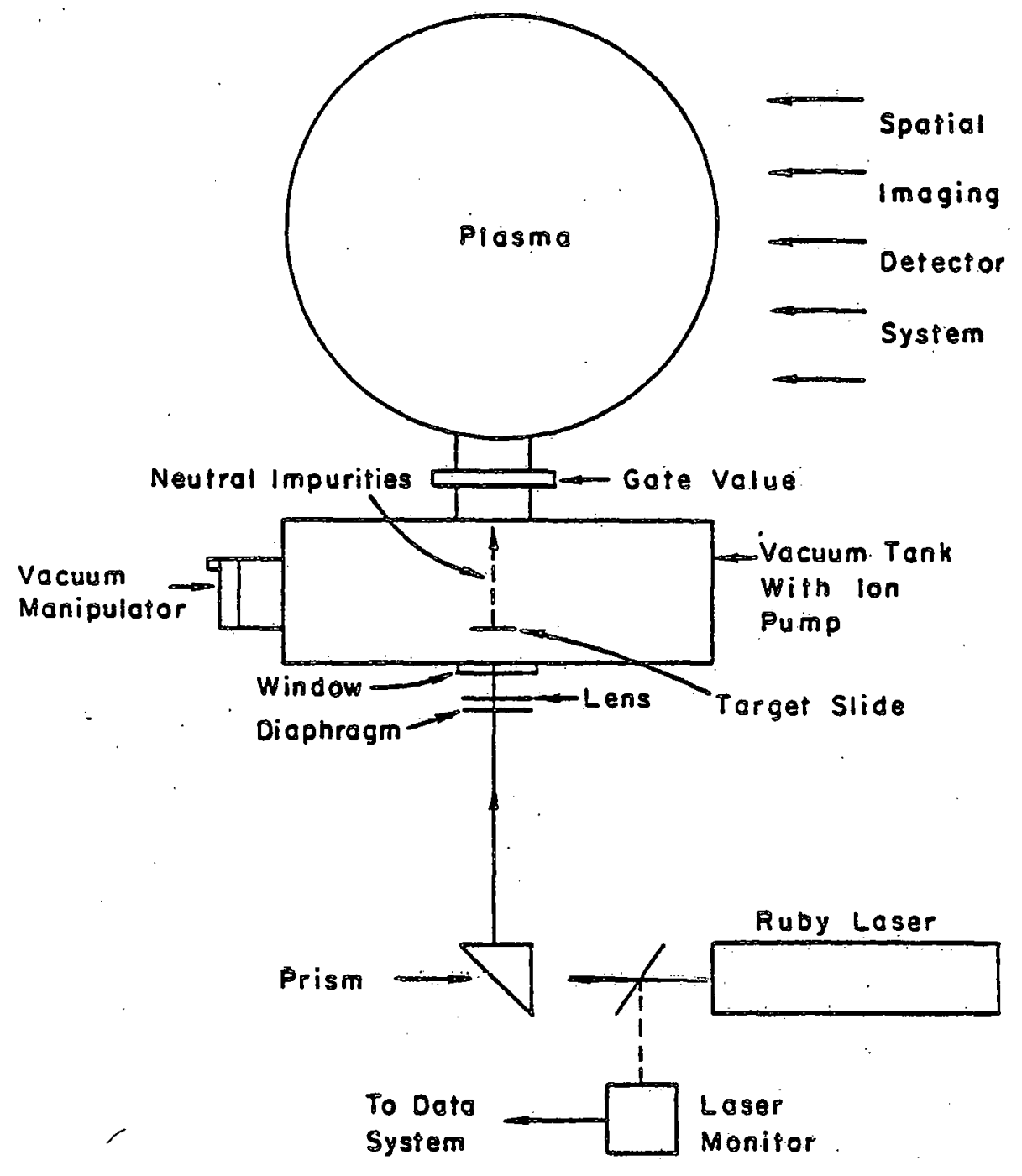

Fig. III-1 
material on the plasma. The results were dramatic; large emissions of MoXIV-424 and $374 \AA$ were observed on the very first injection. It was found (Fig. III-2) that the plasma could be completely terminated by injecting Mo. In Fig. III-2, the sum of the 22 anodes for Mo XIV during this type of discharge is shown; note the large burst of emission and then the plasma disrupts shortly after the injection. Fig. III-3 shows a less severe case when the plasma was perturbed - as evidenced by the current and density. but did not completely disrupt.

Several other impurities were injected, including $\mathrm{Fe}$ and $\mathrm{Al}$, but the element used for the major part of the transport study was Si. Experimentally, it was found that the most reproducible results could be obtained with Si, and at the same time, Si had emissions that could be monitored by the SIDS and a X-ray crystal spectrometer. Si IV (458 $\AA$ ), Si XI (303 $\AA$ ), Si XI (499 $\AA)$, SI XIII (6.65 $\AA)$ and Si XIV (6.18 $\AA)$ were observed under various discharge conditions. In Fig. III-4, the time histories of Li-like (Si XII), He-like (Si XIII), and H-Like (SiXIV) are presented; the brightnesses have been normalized to the peak of each state. The decay of each of the Ionization states should be noted. In Fig.III-5, the inverted spatial profile of Si XIII obtained by the SIDS is presented. The width is approximately $2 \mathrm{~cm}$ FWHM and is found at the radial location $\mathrm{r} / \mathrm{a}=.6$.

In an attempt to compare the experimental results with currently existing theories of transport, a one-dimenoional compuler code has been used to integrate the coupled equations:

$$
\frac{\partial n_{j}}{d t}=-\frac{1}{r} \frac{\partial\left(r \Gamma_{j}\right)}{\partial r}+A_{j} \quad j=1, \ldots 14
$$

where $\Gamma_{j}$ is the diffusional flux and $A_{j}$ are the "source" terms due to 


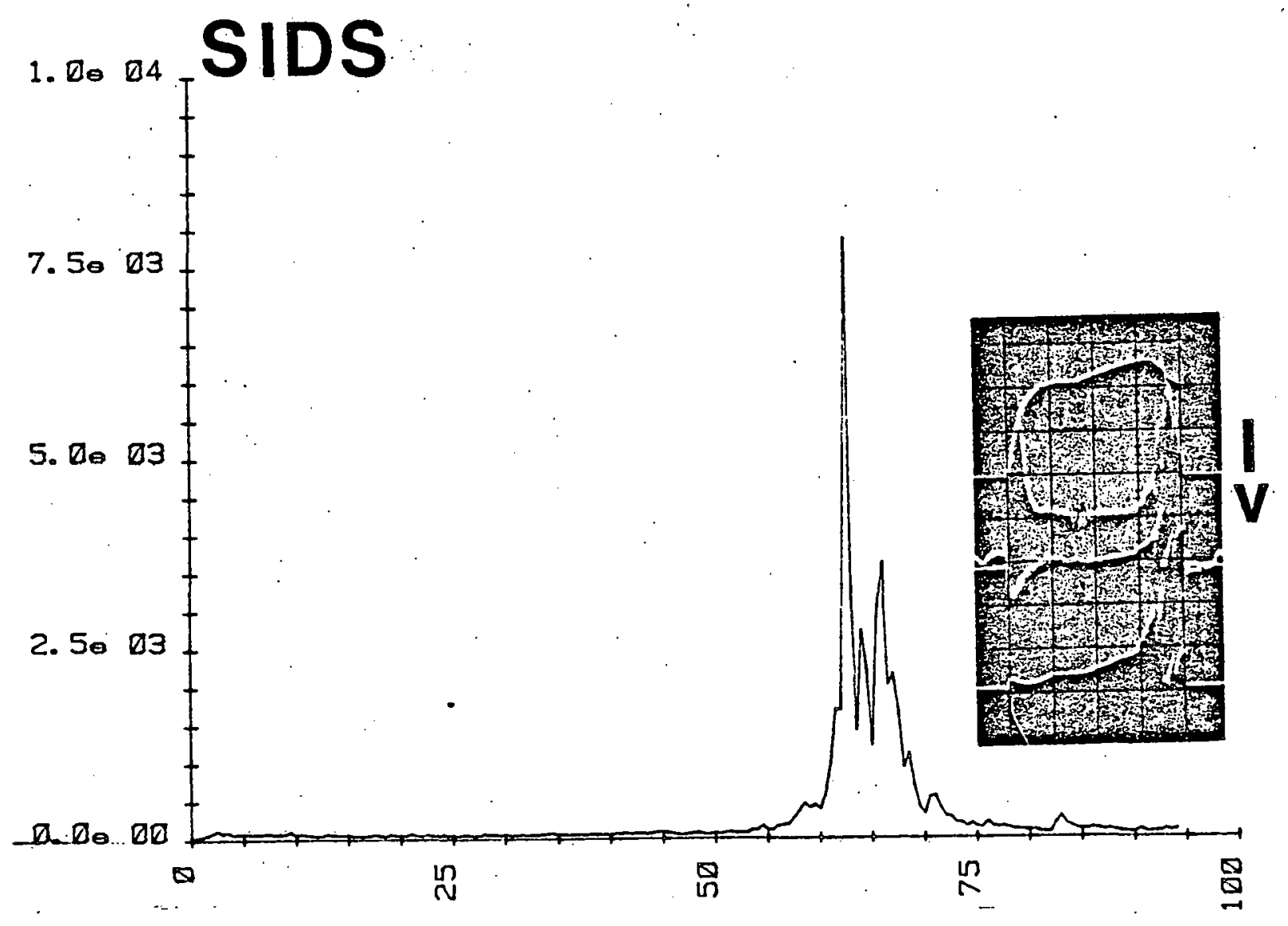

Fig. III-2

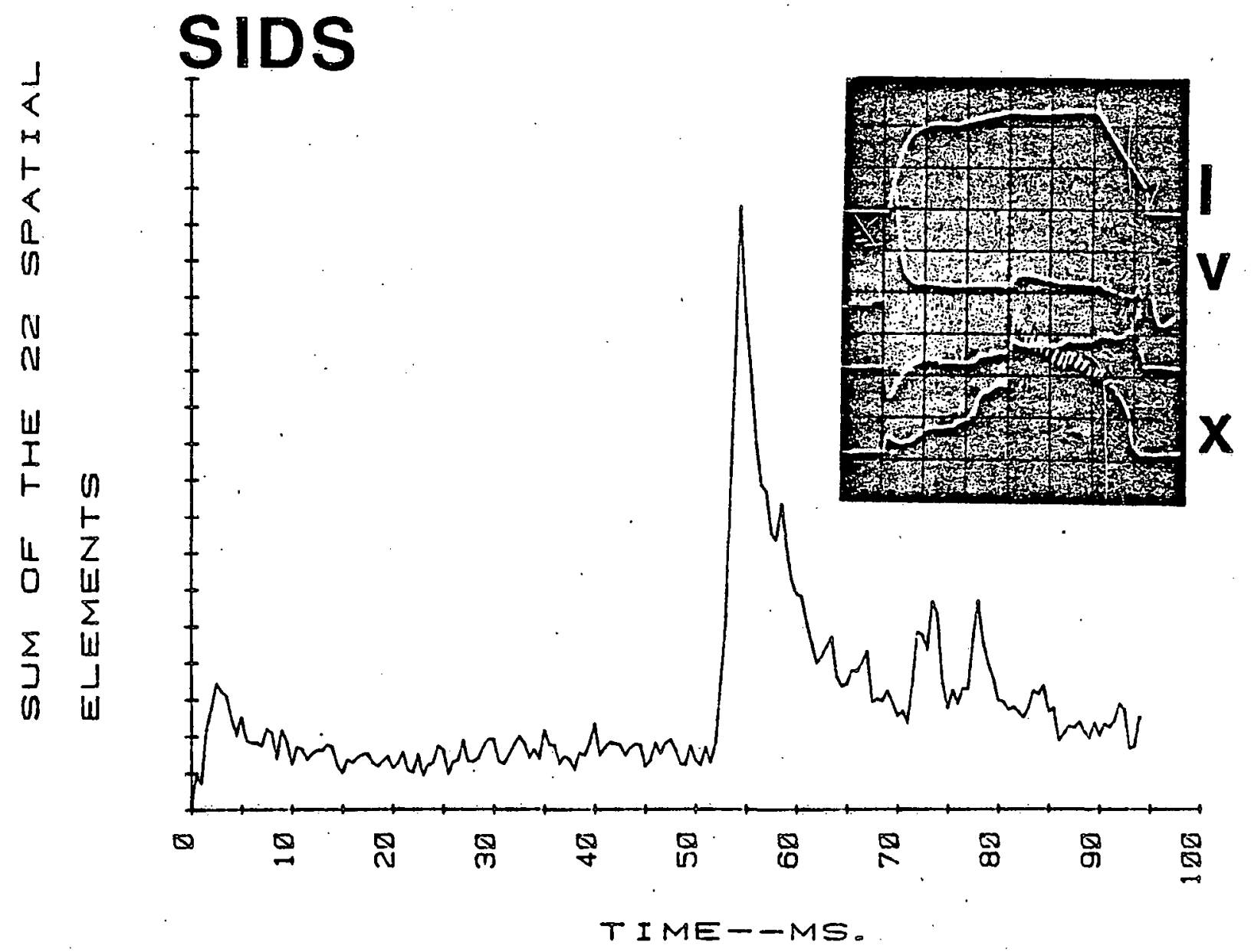

Fig. III-3 


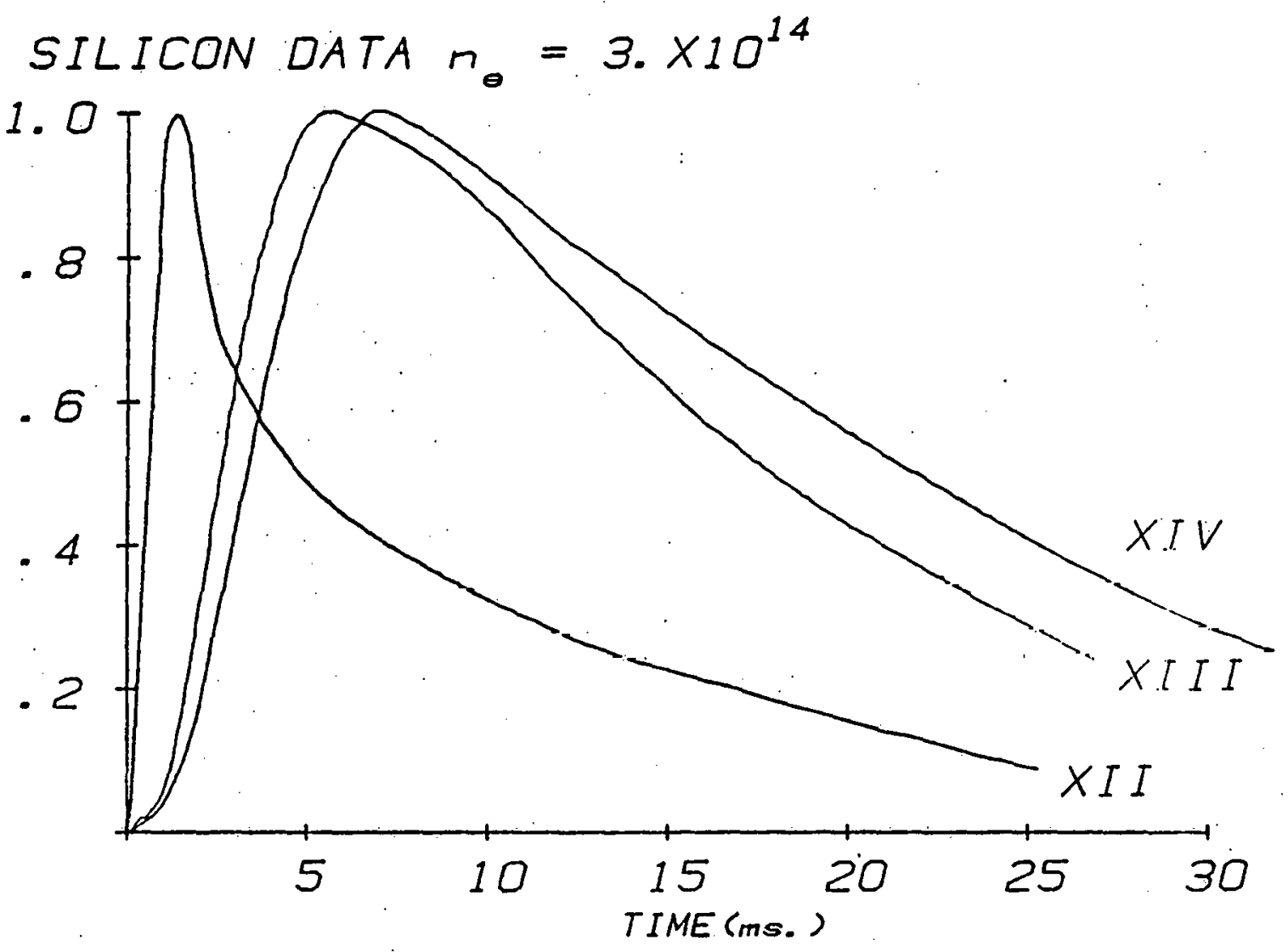

Fig. III-4

SILICON XII Inverted Radial Profile

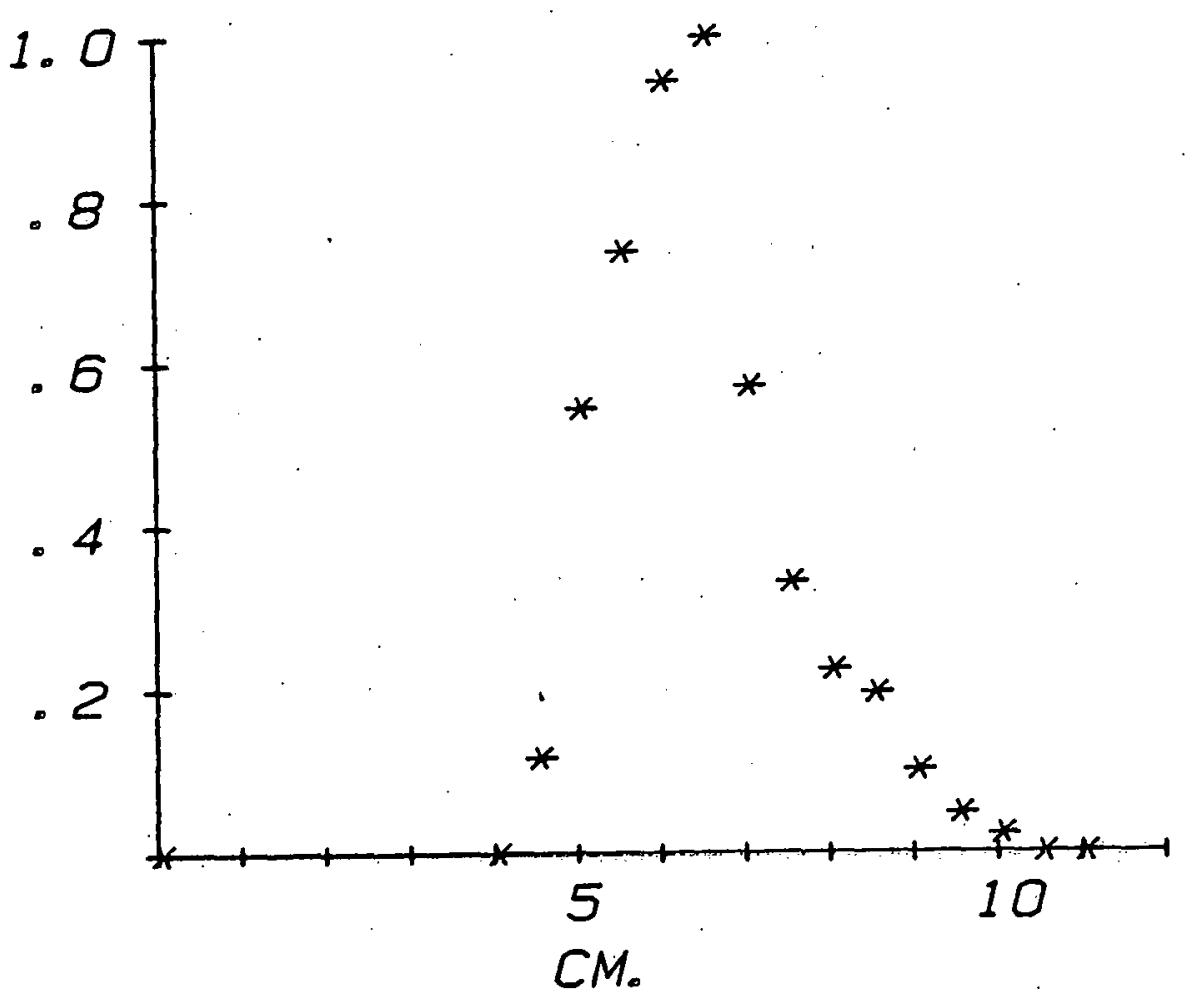

Fig. III-5 
ionization and recombination. In the high-density Alcator discharges, the impurities are in the extreme Pfirsch-Schluter regime, and only silicon-deuter on collisions need to be considered as the effect of the other impurities (Zeff $\approx 1$ ) is not significant. The results of the calculation are shown in Fig.III-6. Note that none of the higher ionization states - SiXIII and SiXIV - decay with a purely neoclassical treatment, indicating the accumulation of impurities in the center of the discharge. SiXII also decays in a different manner than the experimental data. Thus there is a complete qualitative disagreement between a purely neoclassical transport theory and the experimental data. Clearly an anomalous term must be added to the diffusion flux.

The next step was to determine the magnitude of the anomalous transport. Various parameters were varied in the computer model; the results of a factor of 10 enhancement in the neoclassical "self-diffusional" or spreading term (proportional to the silicon density gradient) is shown in Fig. III-7. Note that this agrees quite well with the experimental data except for perhaps the peak in the time histories - this is sensitive to the atomic physics rates. When the computer program is used to model the time evolution of the silicon density, it is found to decay with time. In essence, the silicon is leaving the plasma.

It should be noted that at the electron temperature encountered at the center of the plasma for this type of discharge $(\mathrm{Te}=800 \mathrm{eV})$, the fractional abundances of Si XIII, XIV, and XV are .45, .45. and .1 respectively. The decay of $\mathrm{Si} X I V$ and $\mathrm{XV}$ therefore cannot be due to recombination. In addition, during some higher density, lower temperature discharges, Si XV was not detected; indeed, these are the final ionization states in the plasma. Hence the decay 1s due to the loss of silicon 
SILICON - NEOCLASSICAL

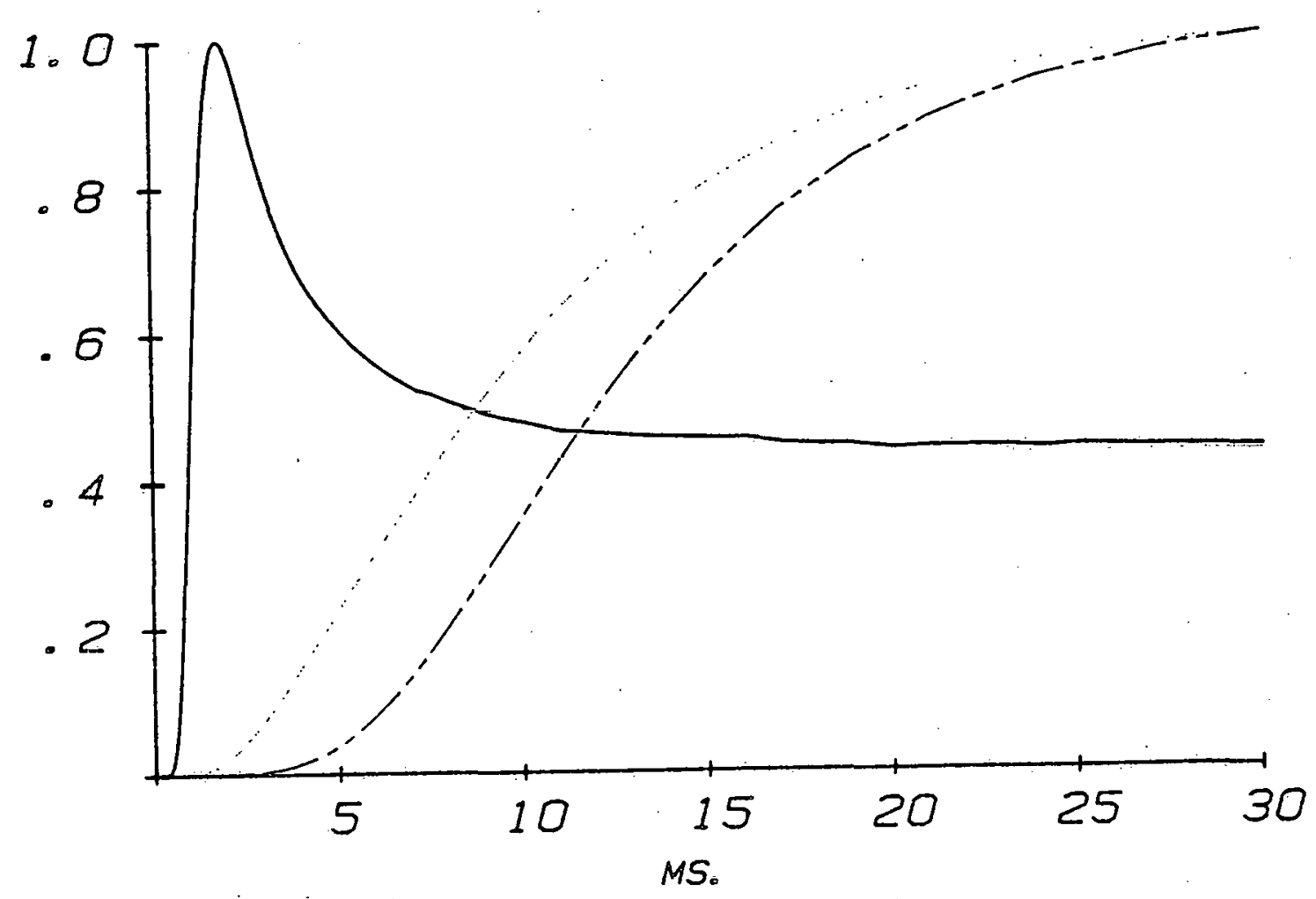

Fig. III-6

SILICON-NEOCLASSICAL+ANOMALOUS

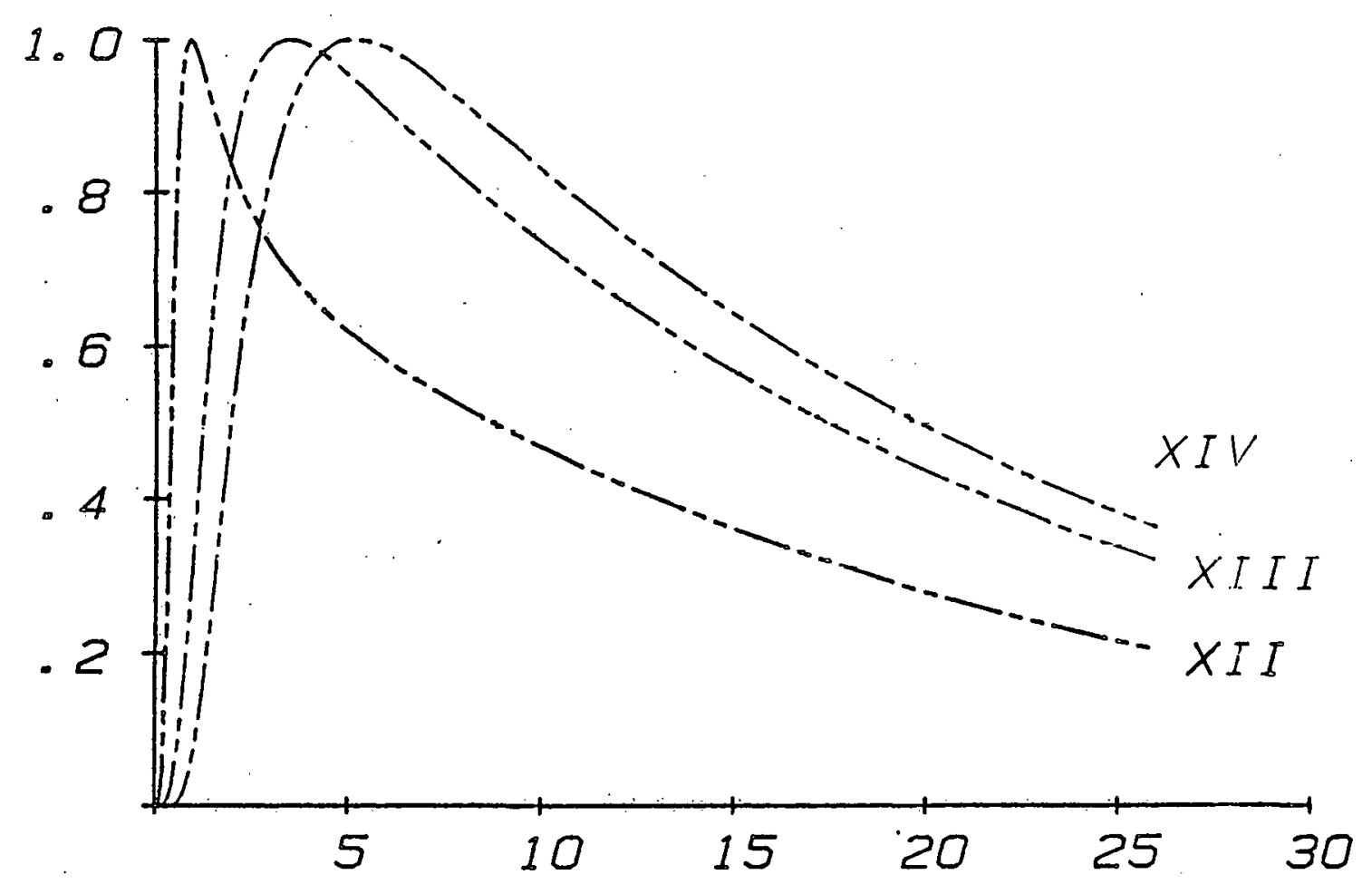


from the plasma and impurities do not accumulate in the center of the discharge. The spatial profile of Si XII predicted by the code with anomalous terms is included in Fig.III-8; it is consistent with the experimental data and only slightly wider than the purely neoclassical case. It would be very difficult to conclude from the spatial profile alone that the transport was anomalous, and even more difficult to estimate the corrections to neoclassical theory. The time histories and the spatial profiles are required. By fitting an exponential to the observed decay of the Si time histories, a silicon confinement time can be obtained. For times shorter than about $10 \mathrm{~ms}$, all three of the ionization states predict a similar confinement time. For longer times, there seems' to be a systematic difference; the Li-like state decays more rapidly, with the discrepancy increasing with the confinement time. This effect is still under investigation, but it may be due to changing profiles in the outer region of the plasma, or it may indicate that the outer region of the plasma experiences a larger degree of enhanced transport.

After studying hundreds of discharges with different parameters; density . $5 \times 10^{14} \mathrm{~cm}^{-3} \leq \mathrm{n}_{\mathrm{e}} \leq 5.5 \times 10^{14} \mathrm{~cm}^{-3}$, current $55 \mathrm{KA} \leq \mathrm{Ip} \leq 230 \mathrm{KA}$, tornidal field $\left(3.6 \mathrm{~T} \leq \mathrm{B}_{\mathrm{T}} \leq 7.9 \mathrm{~T}\right)$ and working gas $\left(\mathrm{H}_{2}, \mathrm{D}_{2}, \mathrm{He}\right)$, certain trends have become evident. In $\mathrm{D}_{2}$ plasmas, with $\mathrm{B}_{\mathrm{T}}=6 \mathrm{~T}$ and $I_{p} \simeq 165 \mathrm{KA}$, the confinement time tends to increase with density, leveling off at approximately $\mathrm{n}_{e}=3.5 \times 10^{14}$. It is found that impurity accumulation does not occur even for these high densities. The confinement time seems to scale nearly linearly with the mass of the working gas. Finally, the confinement increases with increasing plasma current at a fixed density, with the effect becoming more pronounced as the plasma working gas mass is increased. The data is consistent with the scaling of confinement time with q. 
SILICON XII Inverted Radial Profile

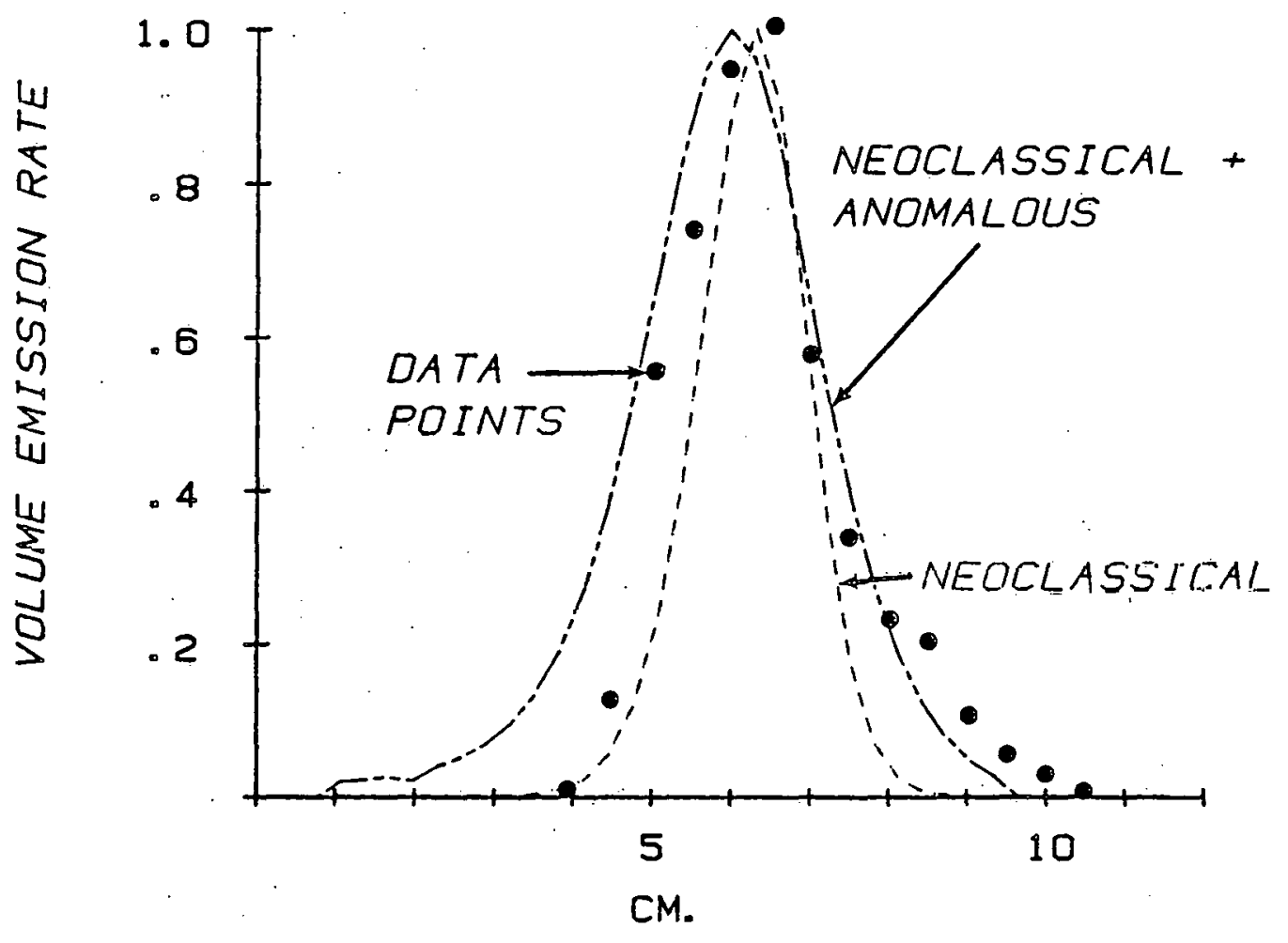

Fig. III-8

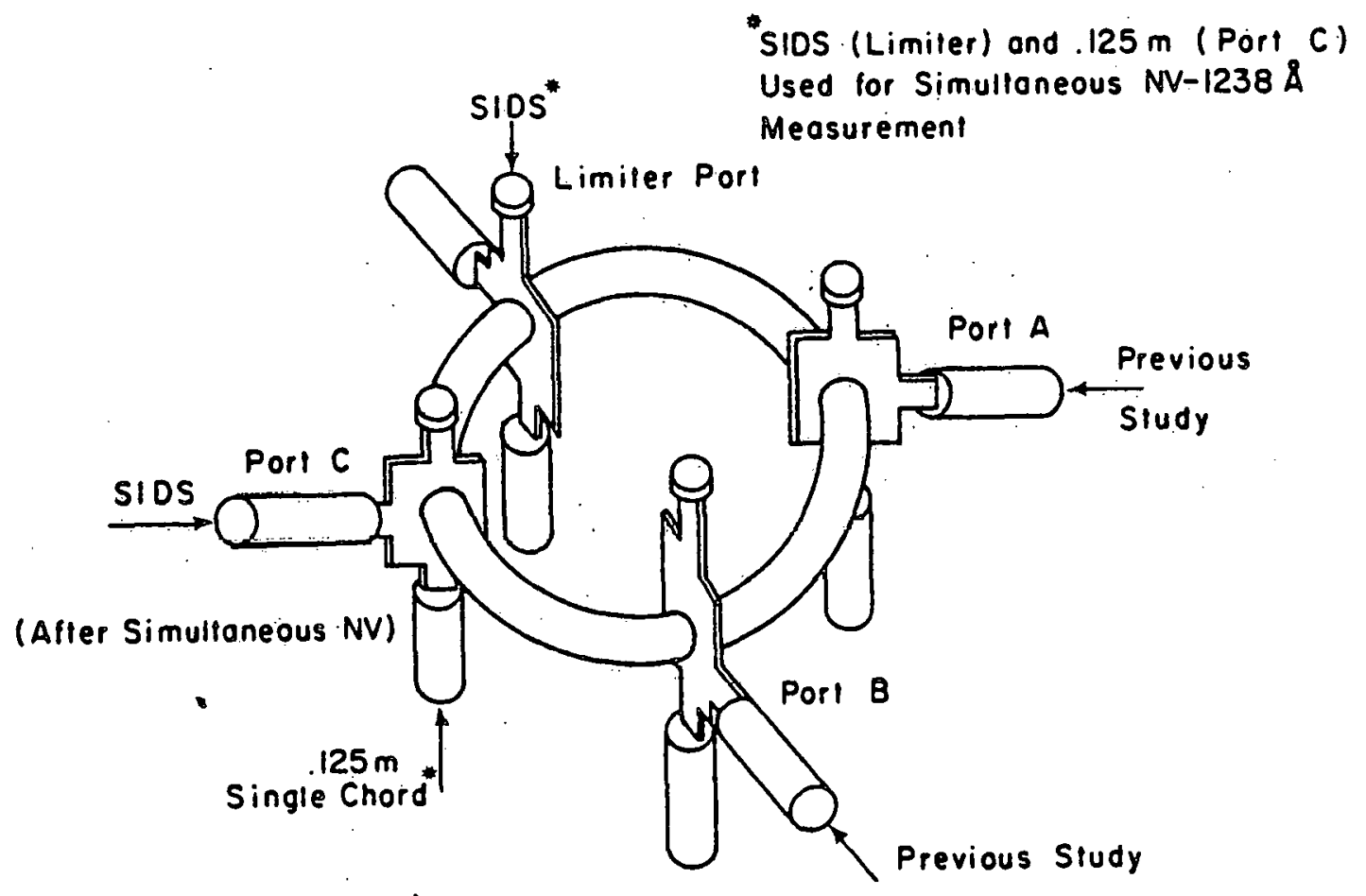

Fig. III-9 
Currently, these scalings and particularly the differences between the confinement time measured at the edge ( $\mathrm{Si}$ XII) and the center (Si XIII, Si XIV) are being studied.

\section{III.A.2.: Toroidal Impurity Transport}

As discussed in the report for the previous contract year, the Spatial Imaging Detector System (SIDS) has been used to measure impurity emissions at the limiter. Because the limiter time histories were different when compared with previous results obtained at Ports A and B in Fig. III-9 a second single-chord .125 m Ebert monochromator was installed at Port C. In this way, the differences could be quantified. To utilize the wavelength overlap of the two instruments, NV-1238 $\AA$ was observed simultaneously.

In Fig:III-10, a single chord of NV from the center of the plasma obtained at the limiter (solid line) is compared with the results at port $\mathrm{C} 90^{\circ}$ away (dashed line). The two brightnesses have been normalized at $80 \mathrm{~ms}$ to accentuate the differences in the time history. The line-average density of this discharge rose to about $1 \times 10^{14} \mathrm{~cm}^{-3}$ at $40 \mathrm{~ms}$, and was constant at this value afterwards. A detailed comparison reveals two important differences:

1. The limiter brightness is greater. Even though it is difficult to measure the brightness at the limiter port because the limiter itself cuts off the field of view in a complicated manner, the limiter brightness is estimated to be a factor of 3 to 4 greater.

2. The decay of the burnout peak is slower at the limiter. While the pnrt $\mathrm{C}$ brightness shows the very sharp peak which is consistent with other measurements at ports $A$ and $B$, the peak at the limiter port decays more slowly. 


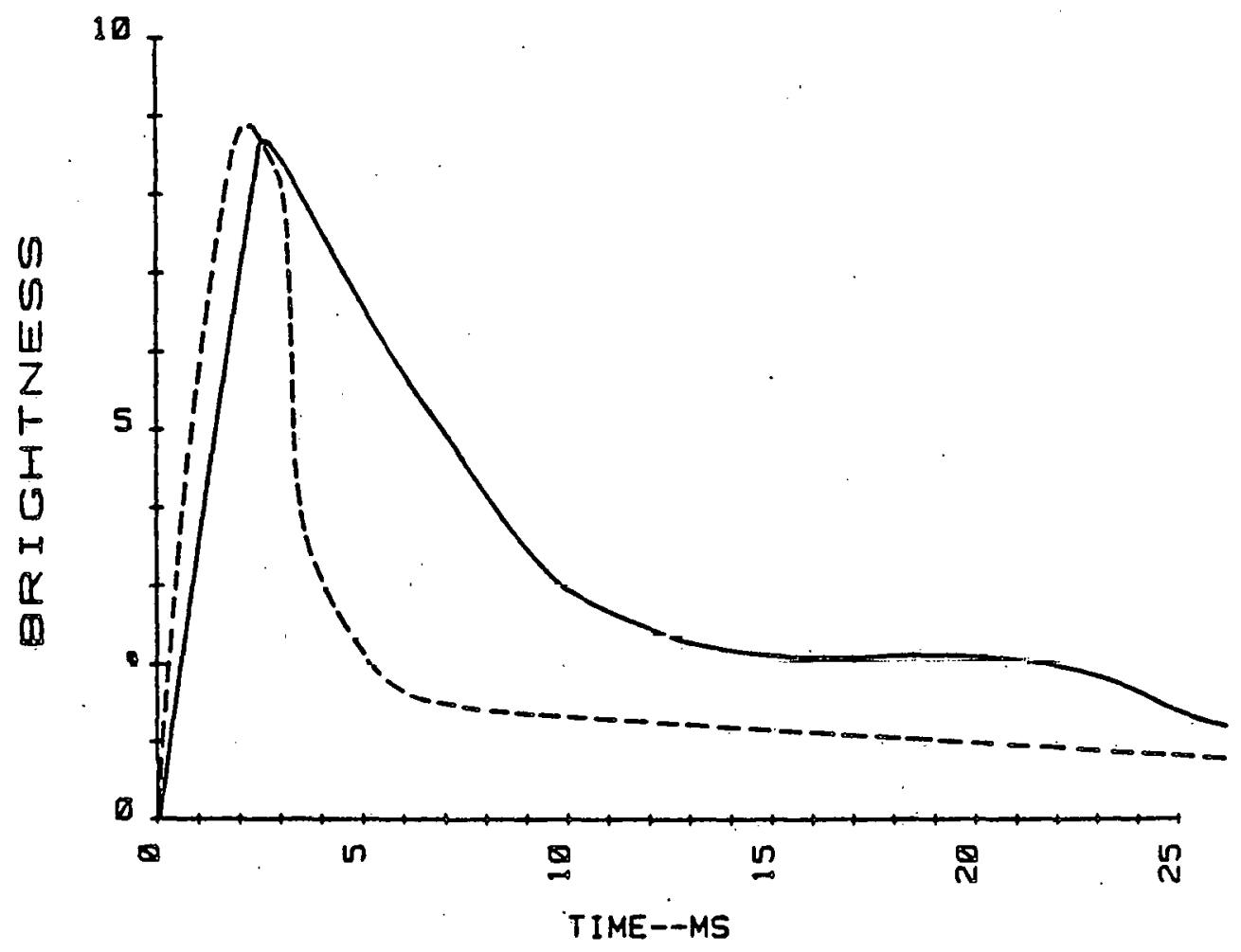

Fig. III-10

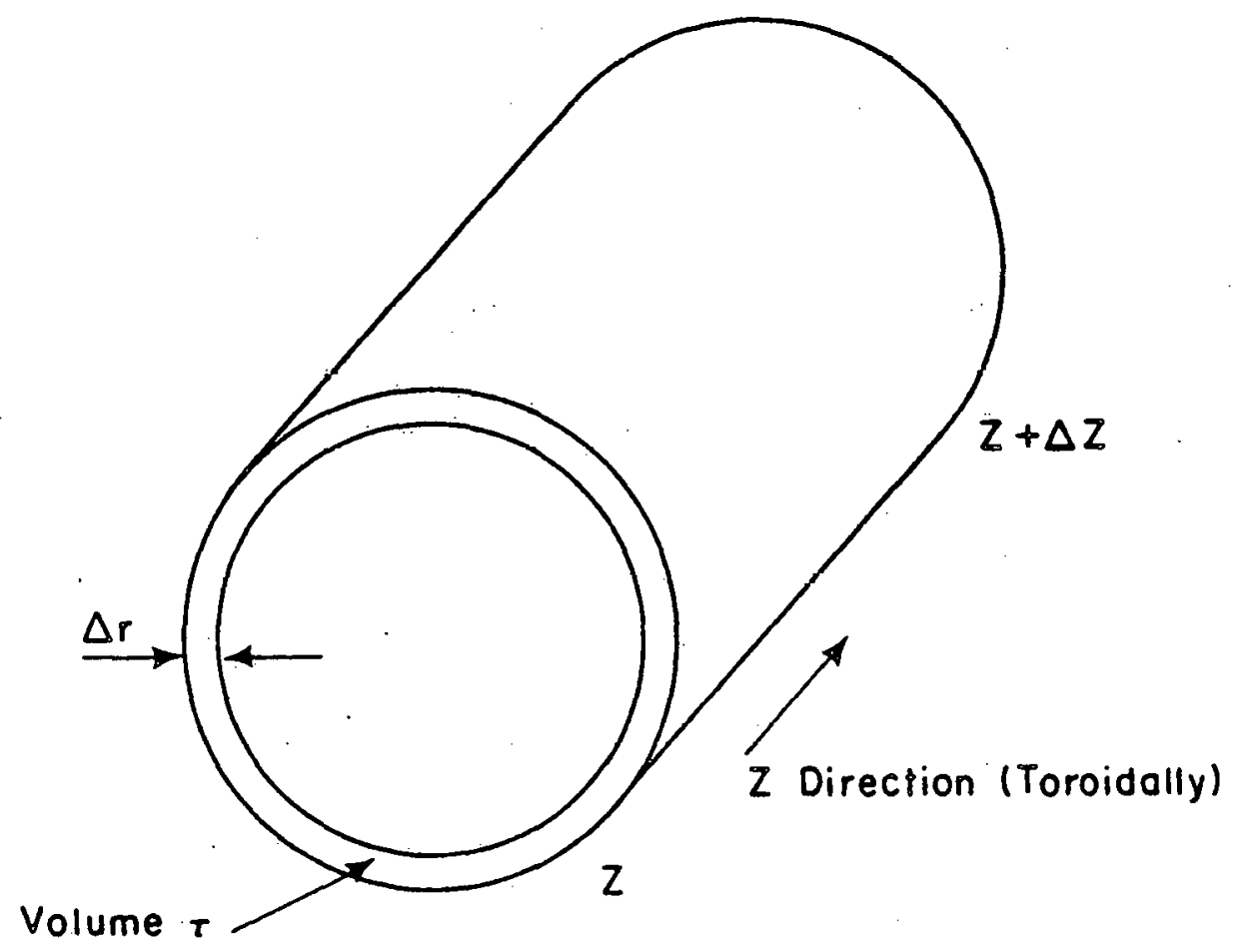

Fig. III-11 
Note that after the low plateau value is reached, the emission does not change substantially and a high density plateau is not evident because the electron density is constant. The time history (but not the absolute brightness) is similar after $30-40 \mathrm{~ms}$. The simultaneous measurement confirmed the differences in the time evolution.

Comparisons of the spatial profiles (also shown in the previous annual report) have revealed erratic behavior in the initial phase of the discharge at the limiter. This is also suggestive of local sources. Later, in the well-developed discharge, the formation of stable shells was predominant, again in agreement with the non-limiter case. Axial symmetry was assumed at the limiter to invert the profiles. This assumption could not be verified because the geometrical obstructions allowed observation of only the outer half of the tokamak.

Of all the observed differences, the most distinctive feature of the ion impurity emission at the limiter is the initial time history. The enhanced brightness is an indication of recycling or a local source at the limiter. Similar results have been obtained from neutral hydrogen emission (6561 \&) at various toroidal locations at Alcator. In the impurity case, there may be some contribution due to charge exchange with impurities at the limiter which is not present at other parts. Several measurements indicate that the neutral density at the limiter is quite high. Charge exchange r.ross sections (which are not well known for these plasma conditions) may be large enough so that this mechanism must be considered.

The explanation of the decay of the burnout peak is a separate question. In an attempt to understand the interaction of the source at the limiter 
and thereby to further understand the observed differences in the emissions, a simple physical model has been used. A local "delta function" source of ions is introduced at the limiter and is traced while it is transported toroidally (parallel to the field lines) and radially (perpendicular to the field lines) through the plasma. The simple geometry of the model is shown in Fig. III-11. A smell volume $r$ of plasma is considered that is located in a shell of width $\Delta r$ and length $\Delta z$. As this volume of plasma moves in the $z$ direction (along the field lines), the number of impurity ions $\mathrm{N}$ in the volume will change because of the inward cross-field flux $F_{r}$. After being transported inward by $\Delta r$, the ions will ionize to a higher state because of the increased electron temperature. The change in the number of ions per unit volume $\mathrm{n}$ is given by:

$$
\Delta \mathrm{n}=-\left(\mathrm{F}_{\mathrm{r}} \Delta \mathrm{z}\right) /\left(\mathrm{V}_{\mathrm{z}} \Delta \mathrm{r}\right)
$$

And in the limit of $\Delta z \rightarrow 0$

$$
\frac{d n}{d z}=\frac{v_{r}}{v_{z} \Delta r}
$$

which has the simple solution:

$$
\mathrm{n}=\mathrm{n}_{\mathrm{o}} \exp [-z / \mathrm{L}]
$$

where $\mathrm{L}$ is the distribution length around the torus $\mathrm{L}=\frac{\mathrm{V}_{\mathrm{z}} \Delta \mathrm{r}}{\mathrm{Vr}}$. This yields a simple physical interpretation of the model: : the ratio of the transport along the field lines to the radial flow across the field lines determines the scale length of the original localized source at the limiter. 
To compute the scale length, $V_{2}$ must be evaluated. This is easily. accomplished with classical collision theory; the parallel collision frequency of the impurity ions with the background deuterium ions must be evaluated. Substitution estimates for $v_{r}(1 \mathrm{~cm} / \mathrm{ms})$ and $\Delta r(1 \mathrm{~cm})$,

$$
L=5.56 \times 10^{6} \frac{T^{5 / 4}}{\mathrm{~N}_{\mathrm{e}}^{1 / 2}} .
$$

Figure III-12 shows a plot of $T$ versus $n_{e}$ for various lengths $L$. Consider the length $L$ in two cases.

1. The initial part of the discharge. Density and temperature profiles in the first 20 - $30 \mathrm{~ms}$ are not well known, but the exact values of $\mathrm{T}_{e}$ and $\mathrm{n}_{e}$ are not necessary. The density at the NV radius is probably in the range $1-7 \times 10^{13} \mathrm{~cm}^{-3}$, and the electron temperature is $\simeq 10-20 \mathrm{eV}$, so the family of curves (Fig. III-12) describing this region is much like the $\mathrm{L}=10 \mathrm{~cm}$ curve. The distribution length is quite short compared to the $85 \mathrm{~cm}$ port-to-port distance.

2. The well-developed discharge is well diagnosed. Doppler widths of the NV line indicate temperatures as high as $70 \mathrm{eV}$. The electron density at the NV radius is in the range of $3-6 \times 10^{13} \mathrm{~cm}^{-3}$. The family of curves describing this region is more like the $\mathrm{L}=150 \mathrm{~cm}$ curve. The distribution length is long in the steady state discharge. 


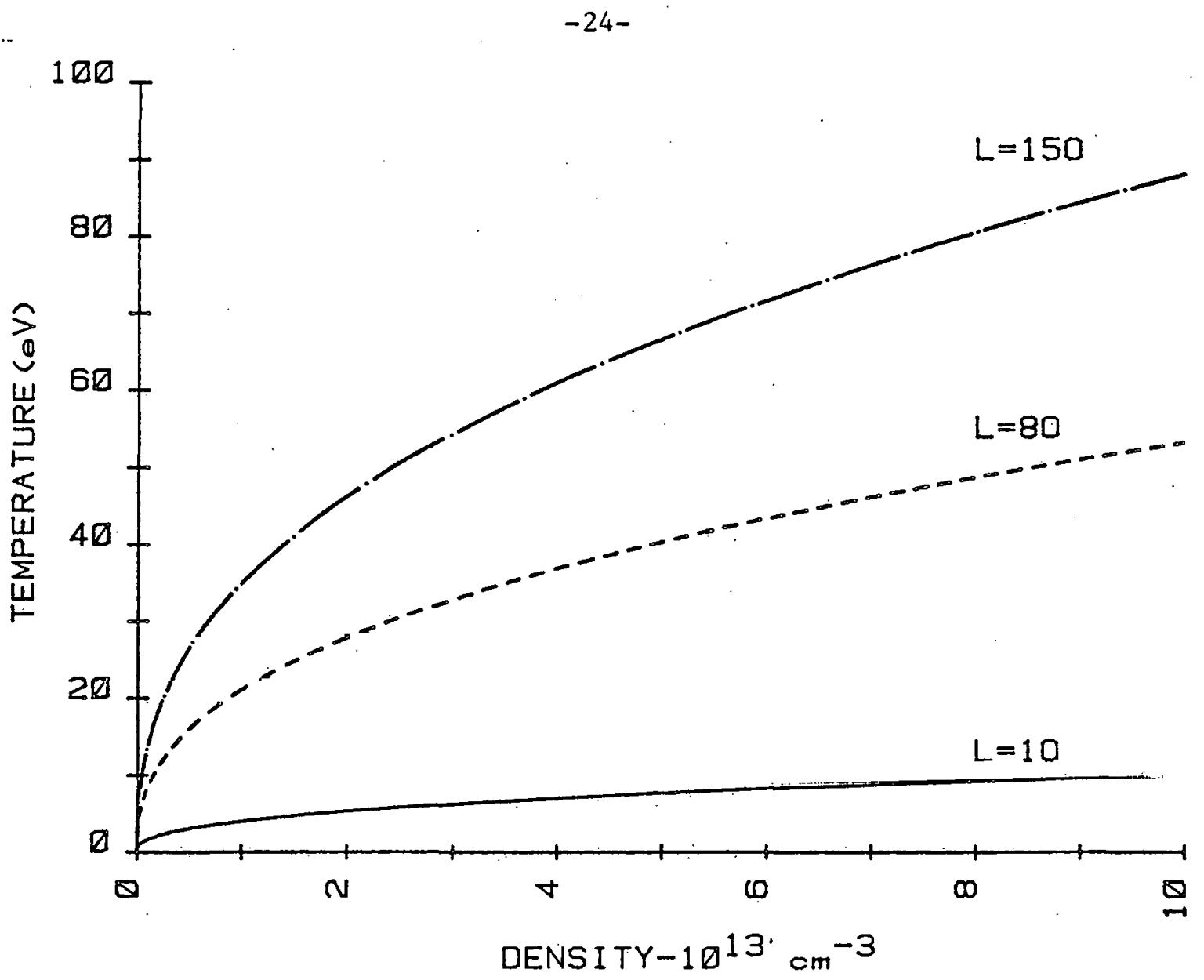

Fig. III-12

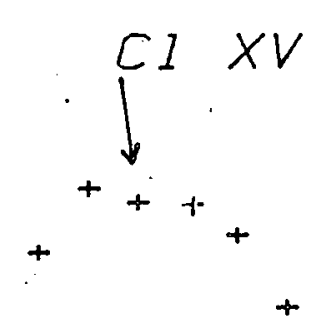

Lower Ionization

+ States

Burflog +
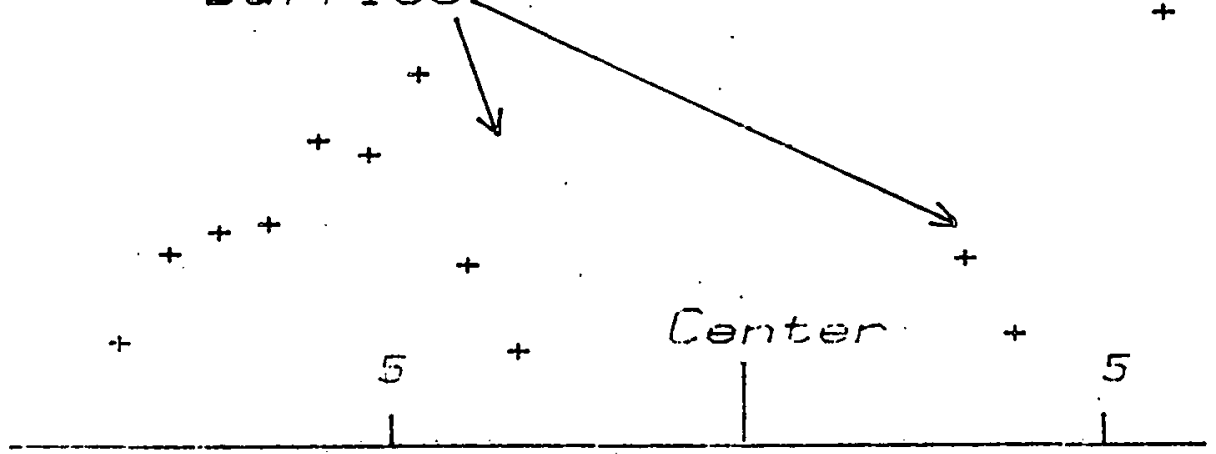

Fig. III-13 
While the model does not treat the detailed interactions in the plasma, it is completely consistent with the main qualitative features of the data - a source at the limiter which is distributed toroidally. The toroidal extent is small at the beginning, and becomes greater as the discharge becomes hotter and more dense. Hence the two locations are qualitatively similar in the steady-state discharge.

III.A.3. Identification of Species by Spatial Profile

The SIDS has been useful for obtaining profiles of emissions with a changing background and highly variable emissions, i.e. MoXIV at the limiter (presented in the previous annual report). In addition, the spatial profile of an ion can be used to distinguish it from other emissions that are at approximately the same wavelength; so ultrahigh spectral resolution is not necessary. A particular example is C1 XIII at $384 \AA$, which is contaminated by other light impurities (c, $N, 0)$ with emissions in the $1 \AA$ bandpass of the SIDS. Fig. III-13 shows a diagram of the brightness profile. Because the ionization potential of $\mathrm{Cl}$ XIII is quite high, it exists in the center of the discharge. The nearly-gaussian electron temperature profile of the plasma forces the lower ionization states to be at the edge of the plasma. In this way, the SIDS allows an identification of a species by spatial profile and wavelength. The baffles shown are plysical obstructions to the field of view and are used to calibrate the absolute radial position. 


\section{III.A.4. Molybdenum Radiative Losses}

A current problem in the design of tokamaks is the choice of material for the limiter. Several different types of material have been tested in tokamaks, and the choice of material for the next generation of tokamaks is not clear. Problems connected with radiative cooling from plasma impurities originating from the limiter is a current topic of investigation. Alcator $A$ and $C$ have a molybdenum limiter and therefore, an experiment was instigated to measure the amount of power radiated to the walls by highly ionized molybdenum.

A one-meter grazing incidence spectrometer was used to measure the brightness of the molybdenum radiation. The instrument has a wavelength coverage of 10 to $450 \AA$ with $1 \AA$ resolution, and was photometrically calibrated between 50 and $450 \AA$ at the National Bureau of Standards SURF II Facility. See section IV.A. for further details.

A spatial imaging bolometer array had measured a power loss of $15 \mathrm{~kW}$ from the central region $(0-3 \mathrm{~cm})$ of Alcator A. ${ }^{1}$ Radiation from high ionization states of molybdenum was suspected resulting from the introduction of this element into the plasma through interaction with the molybdenum limiter. Characteristic central electron temperatures on Alcator A were 800 to $1000 \mathrm{eV}^{2}$ Fractional abundance calculations ${ }^{3}$ assuming the coronal model predict ionization states of Mo XXI - Mo XXXI at these temperatures. Theoretical transition energy calculations ${ }^{4}$ predict a large number of lines between 70-110 $\AA$ for the strong transitions of Mo XXV to XXX. 
Identifying specific lines from highly ionized molybdenum is difficult because of the numerous closely spaced transitions. However, because of the possible large contribution of these states to radiative power loss, this problem has recently received more attention.

Molybdenum XIII, XIV, XXXI and XXXII were identified in the Princeton St tokamak by Hinnov. ${ }^{5}$ Molybdenum XIV, ${ }^{6,7} \mathrm{XXX}, \operatorname{XXXI}$ and $\operatorname{XXXII}^{8}$ have been studied using a vacuum spark and laser produced plasmas. Molybdenum radiation from $\operatorname{TFR}^{9}$ has been studied in the wavelength range of $10 \AA$ to $50 \AA$. Unpublished results ${ }^{10}$ identifying molybdenum transitions from $50 \AA$ to $100 \AA$ on TFR has been the most useful in preparing this report. The brightest lines of molybdenum on Alcator A were located between $65 \AA$ and $100 \AA$. A portion of this region is shown in Figure III-14. The small points represent data taken with a 2.2 meter grazing incidence spectrometer which, however, was not photometrically calibrated. The crosses were taken with the photometrically calibrated one-meter instrument. The data displays a rapid rise at $70 \AA$ and slowly decreases at higher wavelengths. Results on TFR indicate the structure at $70 \AA$ is due tö Lonfzatlun states higher than Mo XVI but less than Mo XXIV. The strongest transition of $\mathrm{XXV}$ is at $75 \AA$, so this line was used for a spatial scan.

Figure III-15 shows the time history of several parameters during a typical shot on Alcator A. Peak plasma current was approximately $140 \mathrm{k}$ Amp, and the line average electron density at $70 \mathrm{msec}$ was approximately $3 \cdot 10^{14} \mathrm{~cm}^{-3}$. The current and electron density reached a plateau stage. around 55 to $75 \mathrm{msec}$. Therefore, this time interval is considered the steady state portion of the discharge. Two different molybdenum signals are 


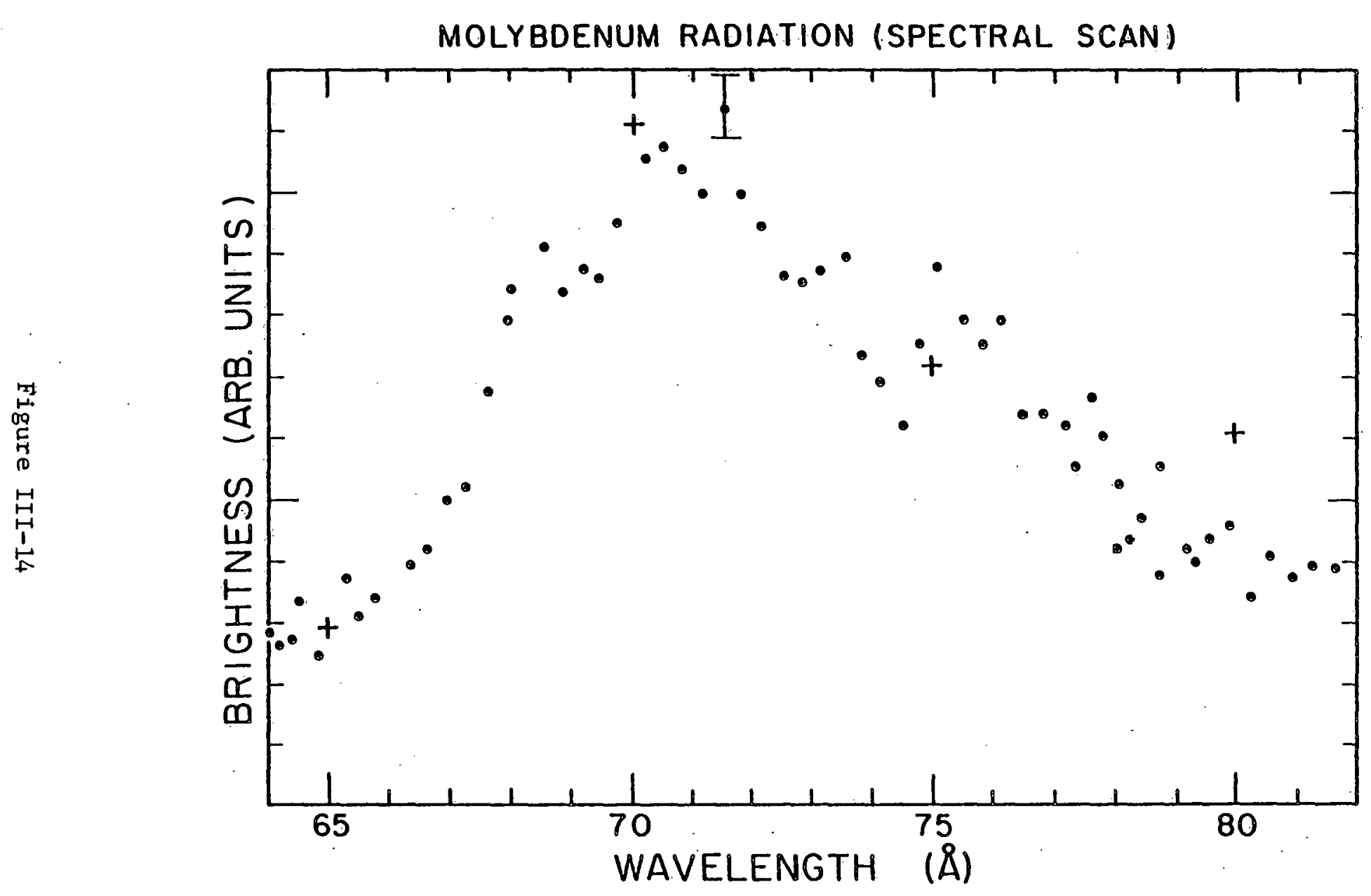


ALCATOR PARAMETERS (NON-INJECTION)

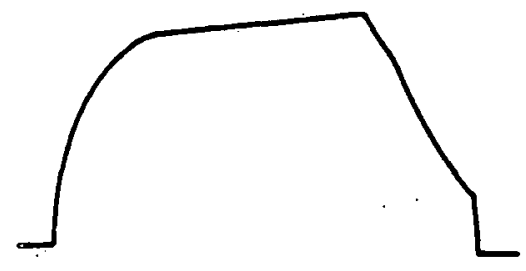

PLASMA CURRENT (150 kA mox.)

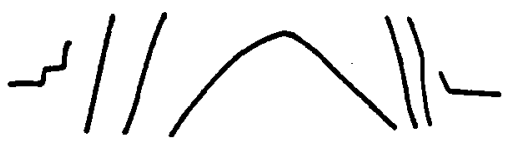

DENSITY

$\left(10^{14} \mathrm{~cm}^{-3} \cdot\right.$ fring $\left.\mathrm{e}^{-1}\right)$

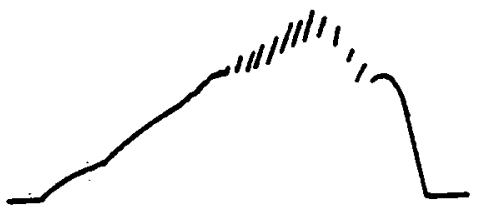

CENTRAL SOFT X-RAY (ARB. UNITS)

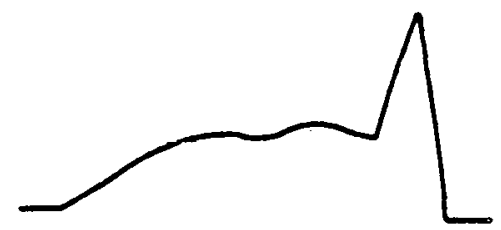

MOLYBDENUM SIGNAL (NO INJ.)

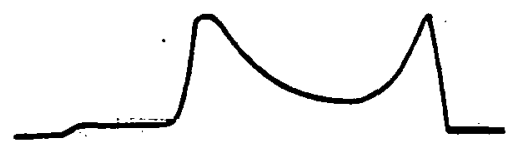

MOLYBDENUM SIGNAL ( ARB. UNITS)

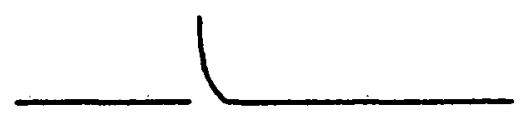

MOLYBDENUM

INJECTION

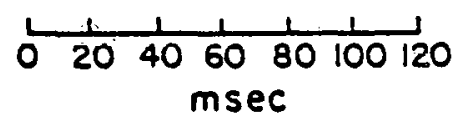


indicated. The molybdenum signal (NO INJ) displays the typical temporal behavior for the emission of molybdenum (56 - $110 \AA$ ) radiation at these electron densities. The signal slowly increases and then reaches a plateau at the steady state portion of the discharge. Either an influx of molybdenum, recombination from higher ionization states, or both caused the spike at the end of the discharge.

In another set of experiments, molybdenum was injected into the tokamak using the laser blow off technique. The spike in the molybdenum injection signal at $45 \mathrm{msec}$ indicates the time the laser fired. The molybdenum signal (INJ) increased and reached its maximum value a few milliseconds after injection. This temporal behavior was consistent throughout the wavelength region $65-110 \AA$ confirming the radiation as molybdenum emission from ionization states considerably higher than Mo XIV. The brightness of the $75 \AA$ radiation (NO INJ) was measured at 10 different chordal positions and inverted using the Abel technique. Figure III-16 shows three dimensional graphs of the Abel inverted data for non-injection and injection spatial scans. Figure III-17 is a linear plot of Figure III-16a for two time periods in the steady state portion of the discharge. Figures III-16a and III -17 indicate the radiation peaked on axis between 65 and $75 \mathrm{msec}$ with a half maximum of $3-4 \mathrm{~cm}$. The volume emission rate was integrated and the wavelengll Epread of 65 $120 \AA$ was folded in to calculate the power radiated by molybdenum. The large spike in Figure III-16b occurred several msec after injection of Molybdenum, then decreased to half of its value in $15 \mathrm{msec}$.

To assure that the line radiation was confined to the range of ionization states of interest (Mo XXI - XXX) measurements were made of transitions 
$75 \AA$ VOLUME EMISSION RATE VS RADIUS AND TIME

(a) NO INJ.

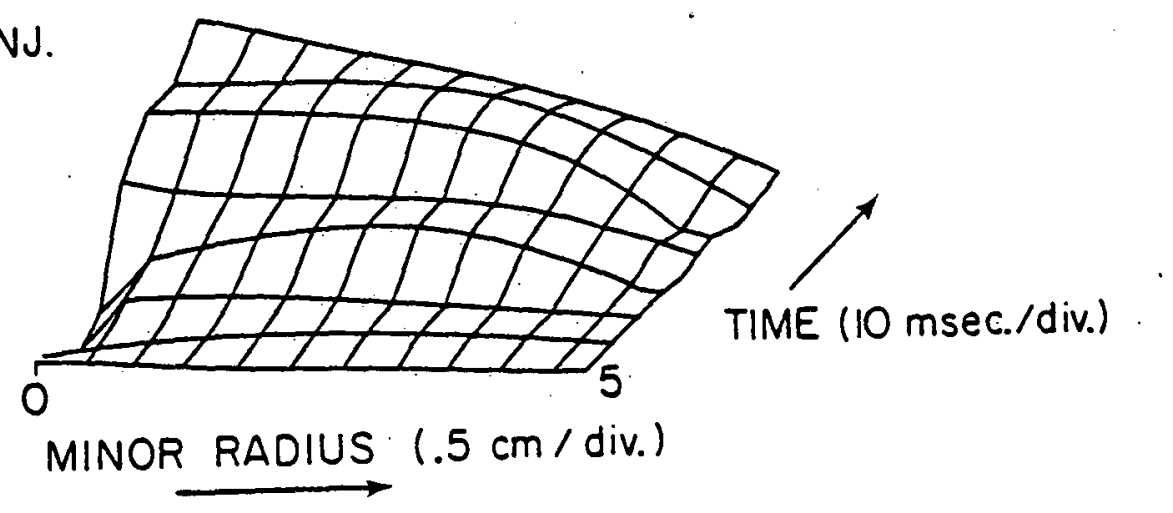

(b) INJ.

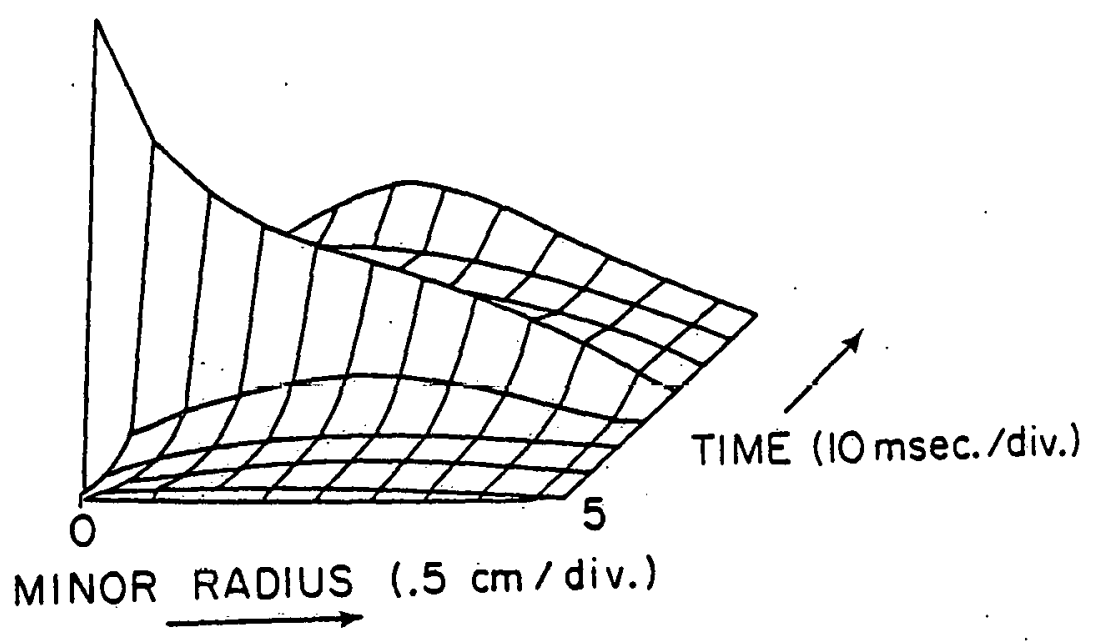


MOLYBDENUM RADIATED POWER DENSITY (STEADY STATE)

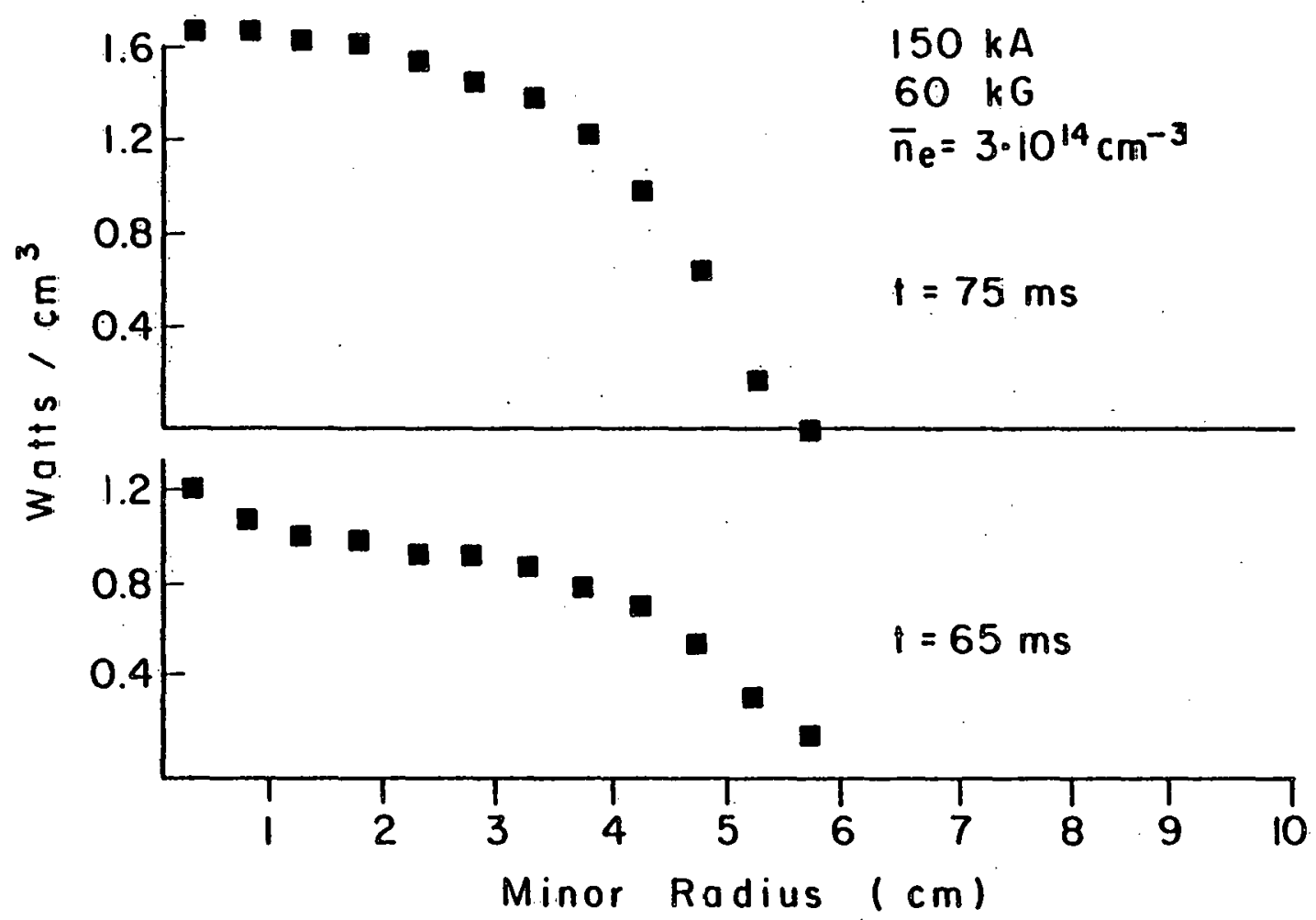


of both lower and higher degrees of ionization. Emission from molybdenum XXXI was an order of magnitude less intense than molybdenum XXV. Those transitions attributed to molybdenum XIV displayed a temporal behavior distinctly different from molybdenum XXV. A series of sharp spikes indicating a rapid rise and burnout differentiated these signals from the more smoothly varying time behavior of molybdenum XXV.

The radiated power loss detected by the grazing incidence spectrometer is displayed in Figure III-18. The temporal behavior indicates that approximately 8 kilowatts was radiated at $55 \mathrm{msec}$, and approximately 25 kilowatts was radiated at $80 \mathrm{msec}$. This is in general agreement with the published values ${ }^{3}$ of the bolometers which measured 15 kilowatts at the peak electron density. The comparison indicates the power measured by the bolometers from minor radius region of $0-3 \mathrm{~cm}$ was due to molybdenum radiation. The local ohmic heating power delivered to the central 0 to 3 in region was approximately $100 \mathrm{~kW}$, therefore the data indicate molybdenum was radiating 15 to $25 \%$ of the local input power at these plasma conditions. 


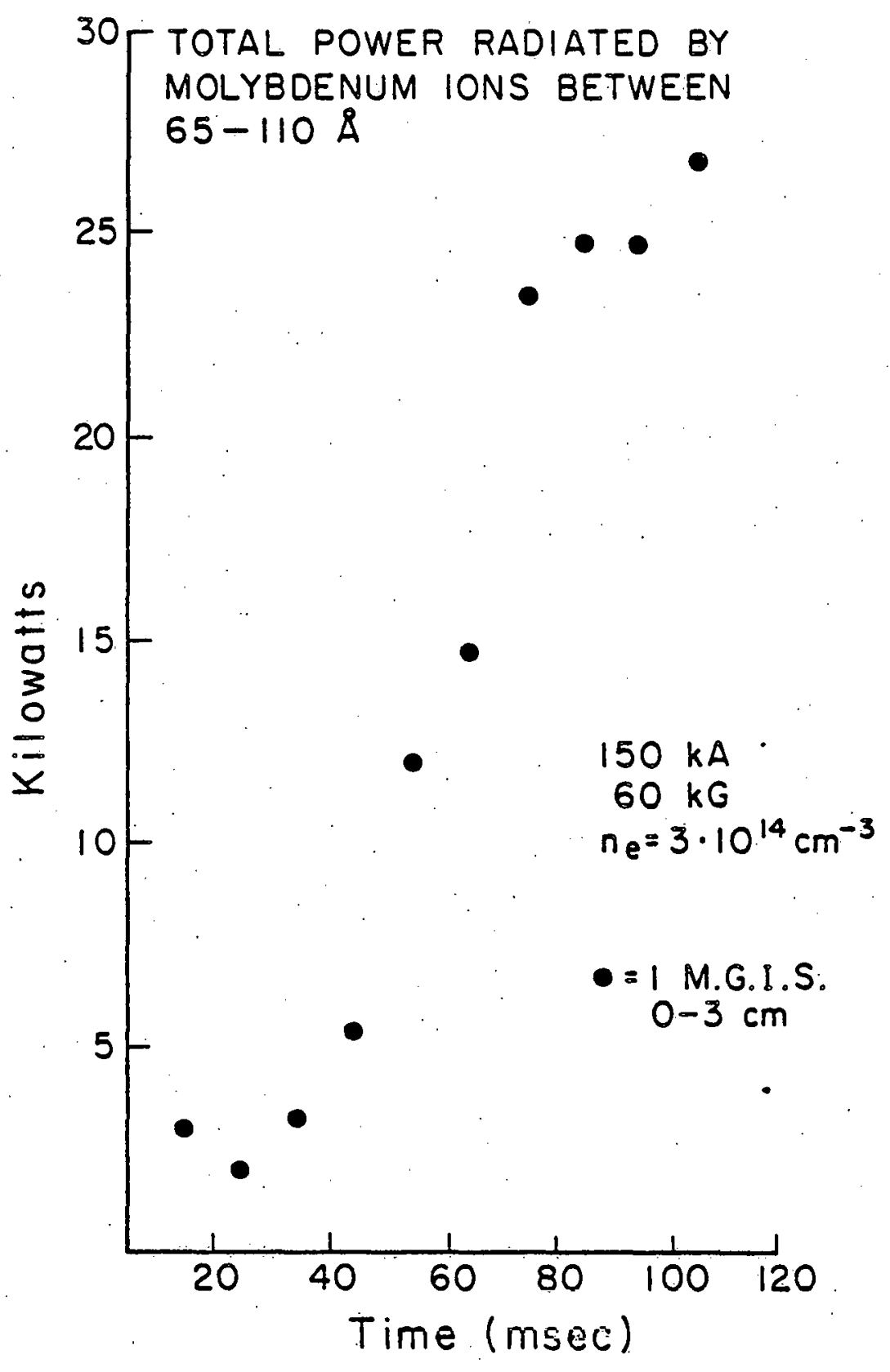

Figure III-18 
III.A.5. References

1. L. S. Scatturo and M. M. Pickrell, PFC/RR-79-8, Francis Bitter National Magnet Lab, MIT, Cambridge, MA.

2. E. Apgar et al, High Density and Collisional Plasma Regimes in the Alcator Program, IAEA-CN-35/A5, Francis Bitter National Magnet Lab.

3. C. Breton, C. De Michelis, M. Finkenthal and M. Mattioli, Ionization Equilibrium of Selected Elements from Neon to Tungsten of Interest in Tokamak Plasma Research, EUR-CEA-FC-948 (1978).

4. R. D. Cowan, Report LA-6679-MS, Los Alamos Scientific Laboratory, Los Alamos, New Mexico (1976).

5. E. Hinnov, Phys. Rev. A, 14, 1533 (1976).

6. L. J. Curtis, A. Lindgard, B. Edlen, I. Martinson, and S. E. Nielson, Physica Scripta, 16, 72 (1977).

7. Joseph Reader, Gabriel Luther and Nicolo Acquisita, J: Opt. Soc. Am., 69, 144 (1979).

8. P. G. Burkhalter, Joseph Reader and Robert D. Cowan, J. Opt. Soc. Am., 67, $1521(1.977)$.

9. J. L. Schwob, M. Klapisch, N. Schweitzer, M. F1nkenthal, C. Breton, C. De Michelis and M. Mattioli, Phys. Letters, 62A, 85 (1977).

10. M. Finkenthal, Private communications. 
III.B. Alcator C

\section{III.B.1. Molybdenum Radiative Losses}

Direct measurements of the flux from high $Z$ impurities in the Alcator $C$ tokamak were begun in late fall of 1979; this work is not yet complete and the results reported here are preliminary. Indications of hollow electron temperature profiles as well as a flattening of the particle confinement time vs. density curve aroused suspicions of a buildup nf heavy element impurities in the hot, central region of the plasma. To study this problem more closely, a photometrically calibrated one-meter grazing incidence monochromator was mounted on Alcator $C$ to determine brightness profiles of relevent high $Z$ impurities such as iron, and in particular, molybdenum.

Highly stripped molybdenum ions, $I \simeq 25$ where $I$ is the degree of ionization, arising from interactions between the plasma and molybdenum limiter are thought to play a significant role in the power balance scheme of the tokamak. Line radiation from these ions presents an efficient mode of kinetic energy removal from the system. As discussed previously, this problem was addressed on the Alcator A tokamak, and it was determined that line radiation from a molybdenum pseudo-continuum in the 65-110 \& range accounted for very nearly the entire power loss measured by the bolometers in the $0-3 \mathrm{~cm}$ region of the plasma.

Transfer of the monochromator to Alcator $C$ was then proposed to invest1gate the same effect. Preliminary observations have shown that, in the $65-110 \AA$ range, the peak brightness, $\simeq 1 \times 10^{14}$ photons $/ \mathrm{cm}^{2}-\mathrm{sec}-\mathrm{Sr}$, was an order of magnitude less than on Alcator A. A striking feature, however; was the broad radial extent of the emission. Half maximum 
at $75 \AA$ was not reached until $\rho=.59\left(\rho=\mathrm{r} / \mathrm{a}_{0}, \mathrm{a}_{0}=17 \mathrm{~cm}\right.$ minor radius $)$. However, the total power radiated was approximately $4 \mathrm{k} \mathrm{W}$. This was a negligible fraction of total ohmic input. The upcoming installation of a pyroelectric device will provide an independent measurement of total power loss and will be compared to those of the monochromator. These initial results prompt other questions. The small contribution in the aforementioned spectral range does not preclude the existence of radiative losses in other wavelength regions. Investigations are now underway to identify other ionization states of molybdenum as well as iron to determine their contribution to power loss. Several spectral lines corresponding to higher ionization states $(I \simeq 31$ ) have been observed. Radial scans of these emissions will determine radiated power. The low brightness of $I \simeq 25$ molybdenum ions may also be an indication of some recycling process which inhibits the production of high ionization states. Examination of low states $(I=6-13)$ near the plasma periphery will lend some insight into the magnitude of any recycling processes.

This ongoing research will investigate other questions as well. Observation for toroidal as well as poloidal asymmetries will be attempted and when coupled with further line identification and medsurement will provide a clearer picture of the role played by high-z impurities in the Alcator C tokamak. 


\section{III.B.2 $Z_{\text {eff }}$ Measurements}

In March of 1978 , the .125 m monochromator was installed on the Alcator C tokamak. The beam line, preslit, and optics were unchanged so that a relative comparison with the brightnesses measured on Alcator A could be made (correcting only for the differences in the minor radii). In this way, the initial measurements of light impurity densities would not be confused by the uncertainties in the atomic physics parameters of the measured emissions, as long as the discharge conditions were similar. (The OVII [1630] and NVI lines used for comparison are not resonance lines and therefore the calculation of the ground state population is subject to greater errors.) The preliminary measurements indicated that $z_{\text {eff }}$ due to light impurities was in the same range as observed on Alcator $A-Z_{\text {eff }} \sim 1.4$ and usually less than 2 for most discharges. The determination of $Z_{\text {eff }}$ was performed in another quite novel manner. The spectral region near $1940 \AA$ was scanned until a region was found that it was free from line radiation. This continuum radiation was measured and then using a precedure similar to that used on the JIPP-II tokamak, ${ }^{1} z_{\text {eff }}$ was obtained. The advantage of performing this measurement in the ultra-violet is that it is less susceptible to errors caused by reflections from the walls of the ports. The extremely sensitive $\mathrm{f} / 5$ optics and pulse-counting electronics of the $.125 \mathrm{~m}$ monochromator made the measurement of the very weak signals possible.

The same measurement in the visible region was carried out by E. Marmar of the M.I.T. staff. For similar discharges, all three methods - UV line emission, UV continuum, and visible continuum all agreed within experimental error. The preliminary conclusion is that for most discharges, the $Z_{\text {eff }}$ is quite close to 1 . 
III.B.3. References

1. K. Kadota, M. Otsuka, J. Fujita, Nucl. Fus. 20, 209 (1980). 
III.C. The Tandem Mirror Experiment

III.C.1. Introduction

This section describes a series of EUV studies on the Tandem Mirror Experiment (TMX) at Lawrence Livermore Laboratories; TMX itself will be discussed briefly in the next sub-section. The following two subsections will describe studies of the center cell and east plug performed to date; the last subsection will discuss a study of transport in the center cell using a 22 chord spatial imaging system which is just being implemented.

The Tandem Mirror Experiment consists of two 2XIIB minimum B type plasma devices - one on each end of a long solenoid. The tandem mirror concept takes advantage of the positive ambipolar potential, several times greater than $\mathrm{T}_{e}$, which develops in a 2XIIB type device between the dense part of the plasma and the end walls of the vacuum chamber. In a tandem mirror, then, the idea is to "plug" the ends of a solenoid electrostatically by means of ambipolar potential barriers created in two mirror machines, one at each end of the solenoid. ${ }^{1}$ In a fusion device, most of the power would then be produced by thermal burning in the central cell.

The geometry of TMX is sketched in Fig.III-19. Power input to TMX is supplied by neutral beam injectors, which maintain dense mirror plasmas in each cell. The high energy plug ions heat the plug and center cell electrons by electron drag. The center rell electrone ionire the gas aud heat the ions supplied to the center cell by gas feed. Thus the system is characterized by $\mathrm{E}_{\mathrm{p}} \gg \mathrm{T}_{\mathrm{e}}>\mathrm{T}_{\mathrm{c}}$, where $\mathrm{E}_{\mathrm{p}}$ is the average ion energy in the plugs, $\mathrm{T}_{e}$ is the electron temperature, which was originally assumed to be the same in both the plugs and the center cell, and $T_{c}$ is the ion temperature in the center cell. A serious question is the degree to 
LOCATION OF .4m MONOCHROMATOR ON TMX

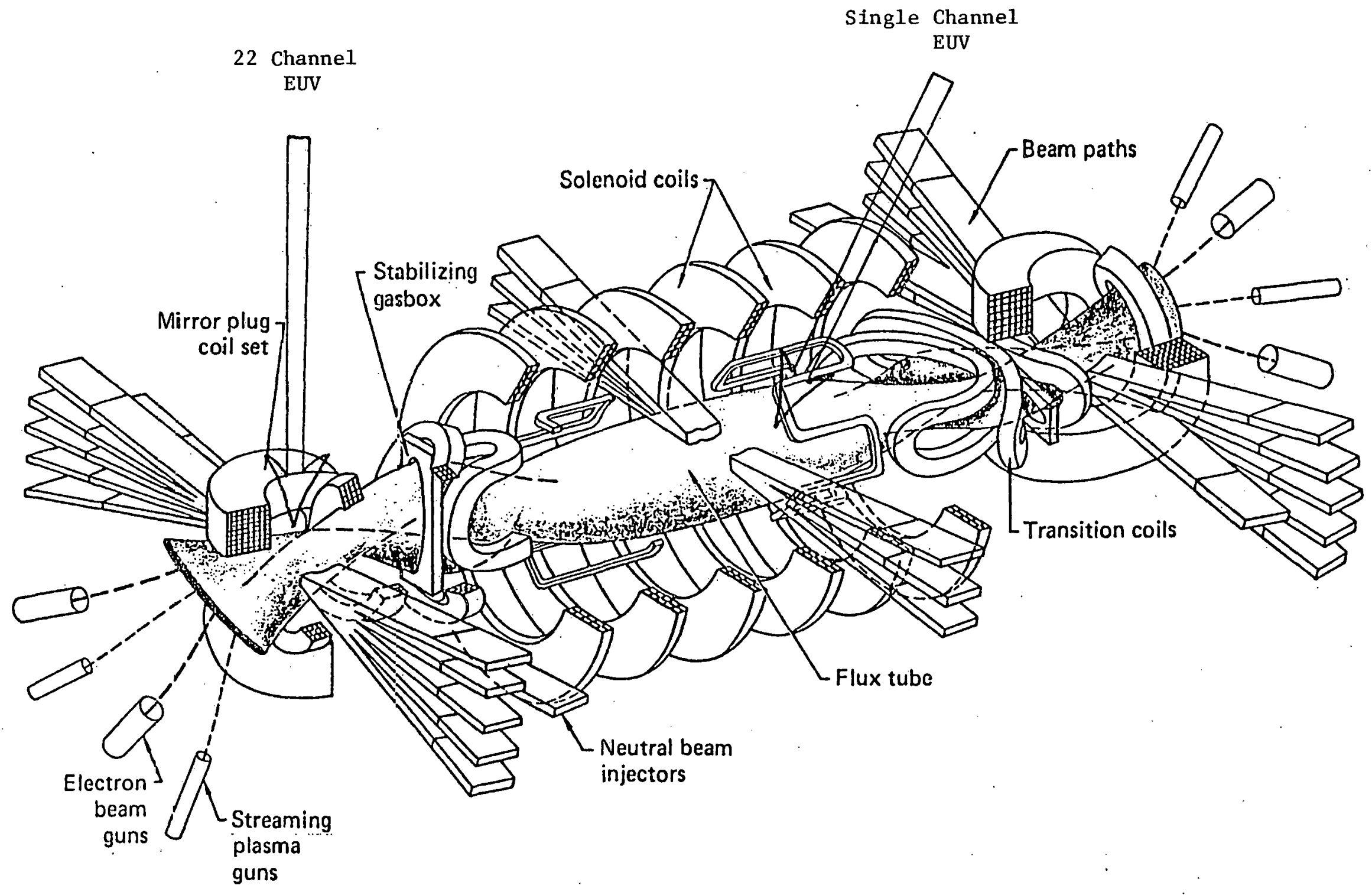


which impurities are transported into and confined in the center cell. To study this problem, a series of EUV diagnostic experiments was begun in the winter of $78-79$.

At present there are two $.4 \mathrm{~m}$ normal incidence monochromators mounted on TMX at the locations marked by the arrows in Figure III-19. The results obtained by these two instruments will be. the subjects of the next two subsections.

\section{III.C.2. The Center Cell Impurities}

The main emphasis on TMX has been to study the physics in the center cell. Therefore, most of our observations have been of that region using a single channel $.4 \mathrm{~m}$ normal incidence monochromator (see previous annual reports). This is the same instrument which was used on Versator and Alcator $A$ at MIT, and on 2 XIIB at LLL.

We have been studying two main issues so far: radiated power loss by impurities from the center cell and electron temperature scaling in the center cell, with variations in plasma parameters. Preliminary results indicate that the major impurities are oxygen, nitrogen, carbon and titanium. The oxygen density fraction is about $0.5-0.7 \%$ which is fairly low. This accounts for a radiated power loss of about $5-10 \mathrm{~kW}$ from the center cell. The brightnesses of nitrogen are about the same as for oxygen, so the power radiated by nitrogen is also $5-10 \mathrm{~kW}$. The brightnesses of carbon and titanium are down by a factor of $5-10$. However measurements by an LLL grazing incidence spectrograph show that the titanium lines are very abundant and emit primarily at short wavelengths (100 $\AA 250 \AA)$. Therefore, titanium could account for another $10-20 \mathrm{~kW}$. Lyman $\alpha(1215 \AA)$ 
is very bright and radiates about $5 \mathrm{~kW}$ alone. These species, then, account for $25-45 \mathrm{~kW}$ of radiated power, about $25 \%$ of the total power lost from the center cell.

Determining a value for electron temperature from spectroscopic data is very model dependent. Very little radial information exists for this plasma, and the instrument was able to view only a central chord of the center cell. Thus, only a rough value can be estimated. However, the data does indicate decreases or increases in electron temperature with TMX parameters by the change in the relative densities of the individual ionization states. For instance, the electron temperature appears to increase with increasing center cell magnetic field. As shown in Figure III-20, the high ionization states of oxygen are much more abundant at $1 \mathrm{kG}$ and $2 \mathrm{kG}$ than at $0.5 \mathrm{kG}$ in the center cell. Likewise, laser scattering data in the end plug also shows an increase in $\mathrm{T}_{\dot{\mathrm{e}}}$ with increased magnetic field. Increasing the neutral beam current in the end plugs increases the center cell electron temperature up to 100 amps of injected beam current per plug (see figure III -2I). (Above that current, the total oxygen density continues to rise but no-significant increase in $T_{e}$ is indicated.) For a brief time, neutral beams were injected directly into the central cell plasma. Figure III- 22 shows a clear increase in electron temperature while the center cell neutral beams are on. The brightnesses increase in the high ionization states while the low lonization state actually appears to have burnt out. This effect is most dramatic when the neutral beam current in the end plugs is low ( 50 amps/plug). Finally, when an electron beam was injected axlally through the machine (to suppress RF fluctuations and increase confinement times), the EUV spectrometer showed a slight increase in 


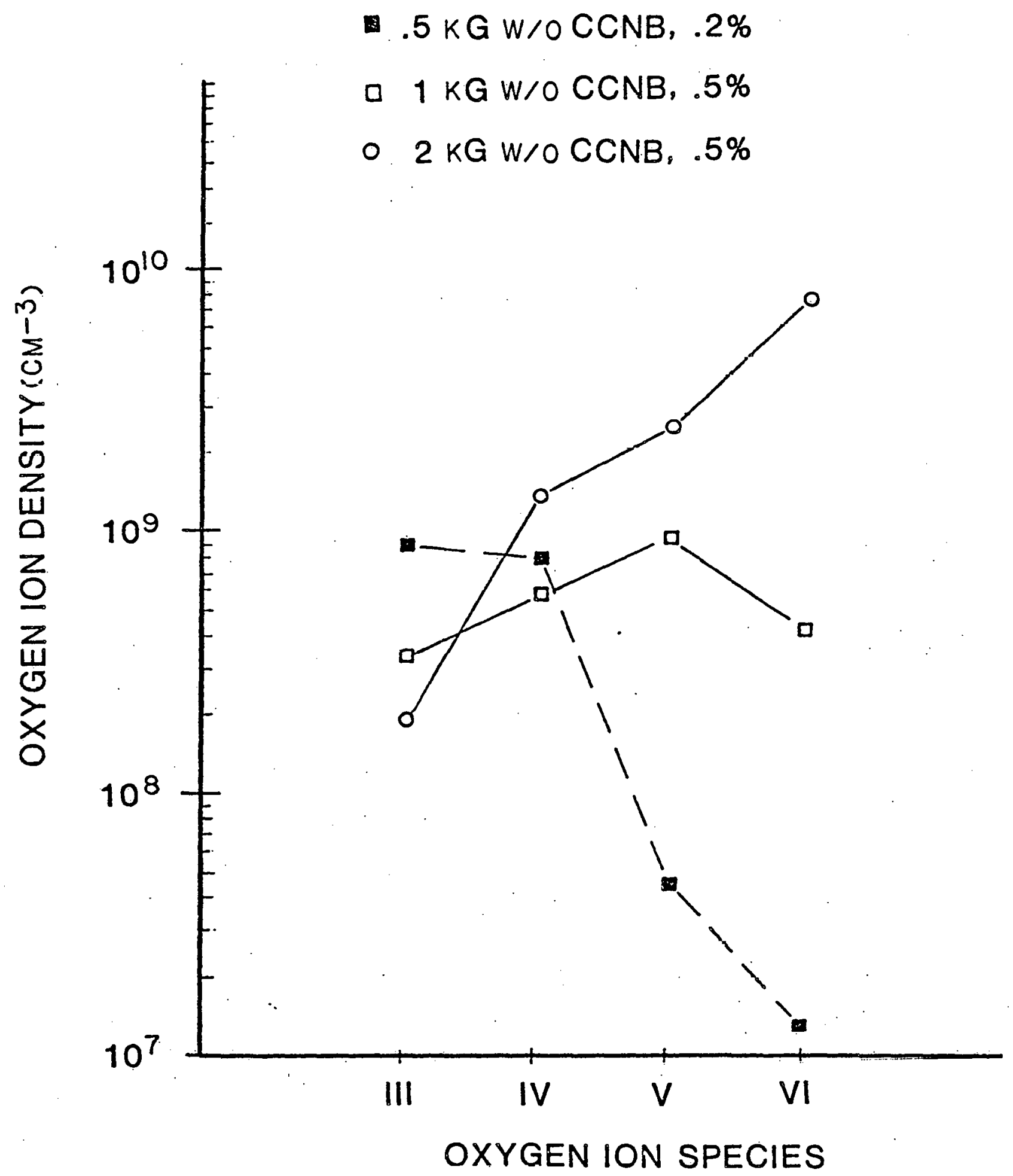

Fig. III-20. The density of oxygen ions for several values of center-ce magnetic field. 

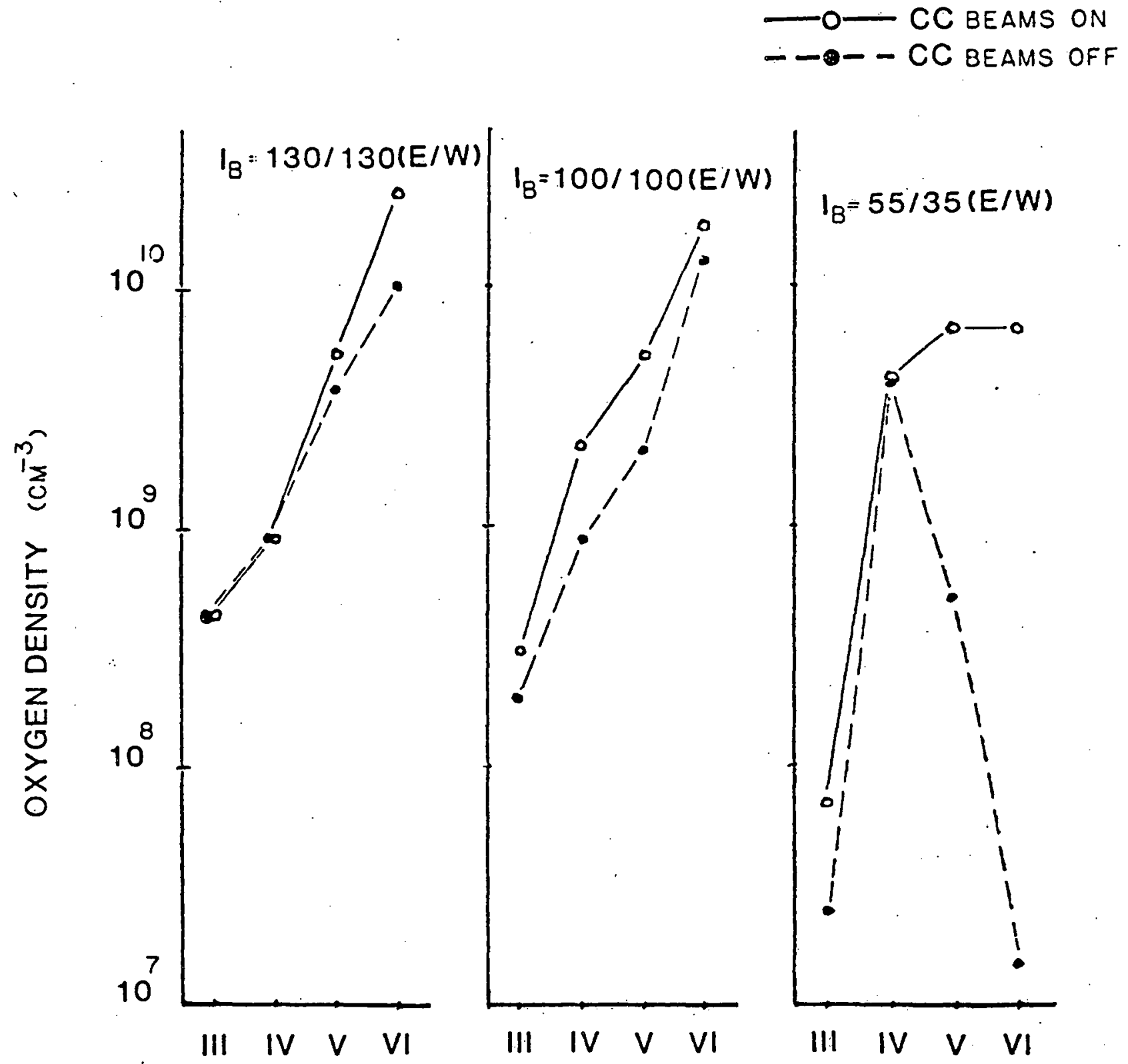

OXYGEN ION SPECIES

Fig. III-21. The density of oxygen ion species in the center-cell for several plug neutral-beam currents, with center-cell beams on and off. 
11/5/79

$B_{C}=2 k G$

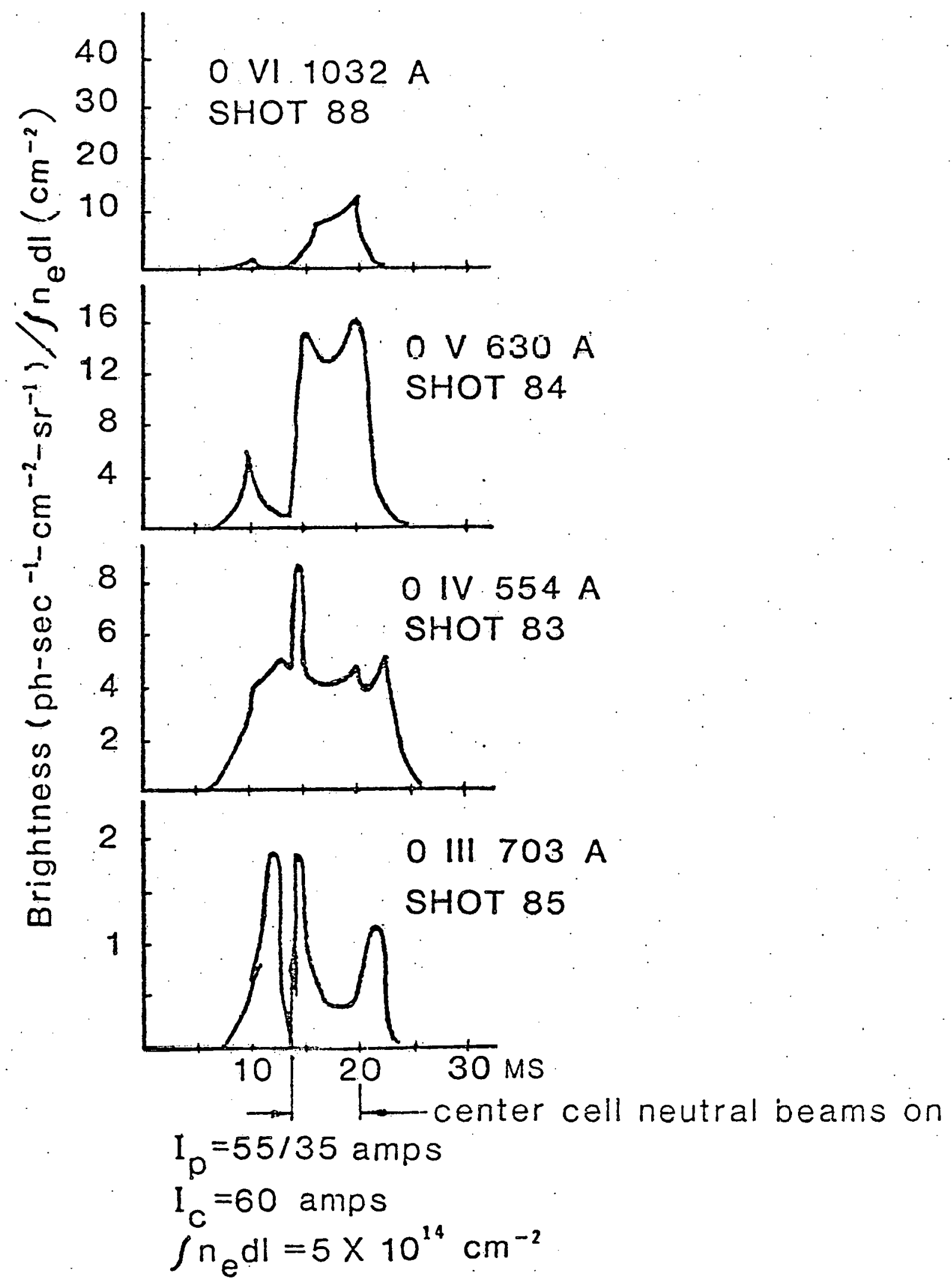

Fig. III-22.. Evidence of increasing $T_{e}$ during center-cell neutral-beam injection. Low ionization states of oxygen burn out, while higher ionization states increase when center-cell neutral-beams are turned on. 
center cell electron temperature. In general, other diagnostics noted little effect on machine operations due to the e-beam although the diamagnetic loops showed a slight increase in ion energy in the center cell. 
III.C.3 Spatial Imaging of East End Cell

Recently, LLL acquired a spatial imaging detector system (SIDS) similar to the Hopkins instrument discussed in previous annual reports. This instrument was briefly mounted on the east end plugs to perform a set of preliminary studies since extensive spectroscopic information had been obtained on the similar 2XIIB plasma. Immediately evident was the fact that the oxygen brightnesses on the TMX plug were much lower than those on 2XIIB, the oxygen impurity density in the TMX plug is about $0.1-0.2 \%$, down an order of magnitude from the 2 XIIB results. This is a rather dramatic change, and further work is indicated. The other interesting result is that the oxygen density is lower in the end plug than in the center cell: .1-.2\% as .5-.7\%. This is evidence of electrostatic plugging due to the ambipolar potential described in subsection II. A multiply ionized impurity species is expected to be confined much more effectively by a given potential than a singly ionized species, resulting in a higher impurity density in the center cell than in the end plugs.

Fig. III-23 shows three spatially resolved EUV emissions from the east end cell. Several features are prominent in the complex behavior of the EUV emissions. The cool start-up deuterium plasma which provides a target for the neutral beam injectors is injected at a radius of about $6 \mathrm{~cm}$ from 5 to $10 \mathrm{~ms}$ into the shot. Its presence is clearly apparent in the deuterium emissions. The higher ionization states (OVI) tend to emit near the interior of the plasma while the lower ionization state (Ti IV) emits near the edge giving evidence for shell structure in the plug plasma. The asymmetry in the emission may indicate localized impurity sources. 
BRIGHTNESS - VS RADIUS AND TIME

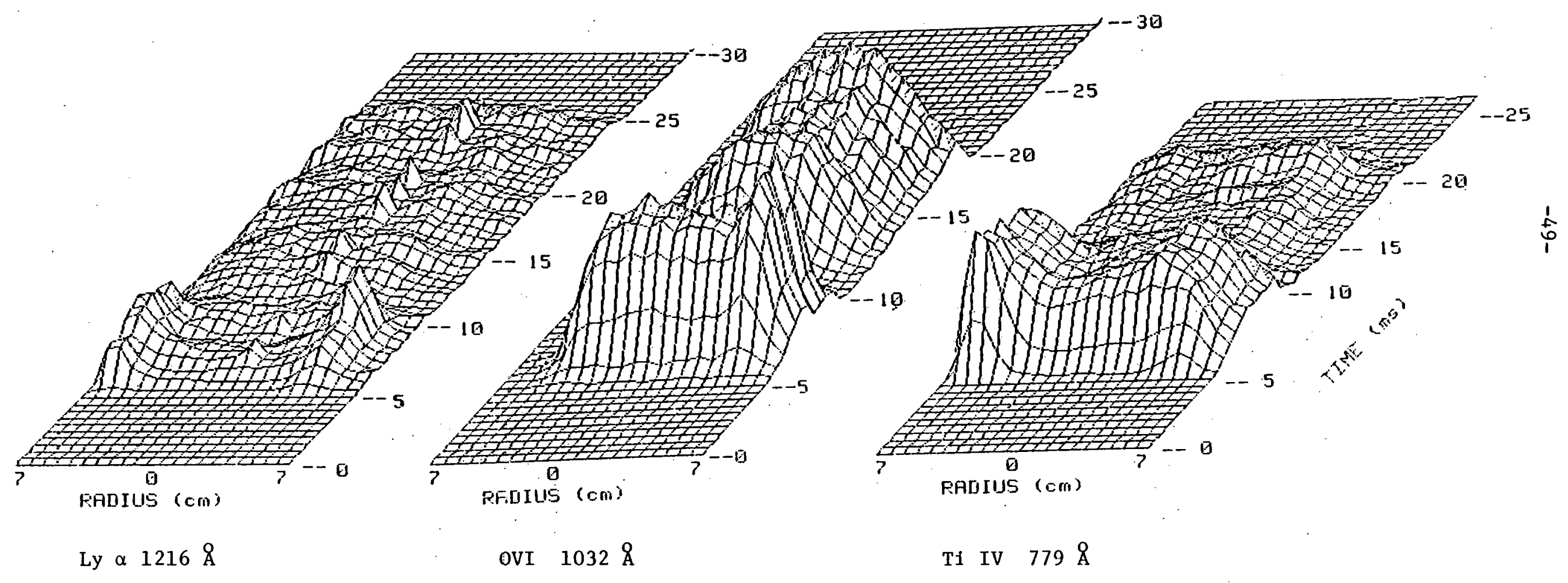


III.C.4. Spatial Imaging of Center Cell

To date, very little spatial information has been obtained by any diagnostic on the TMX center cell plasma. A SIDS spectrometer has been installed on the TMX center cell for this purpose. In addition, the original SIDS spectrometer, used on Alcator A as described in a previous section, has been shipped to TMX. With a spatial imaging detector system on one end pluy, another on the center cell, and the single chord instrument on the other end plug, it will be possible to monitor all three plasma regions of TMX simultaneously. This will greatly increase our ability. to study TMX, in that the total number of plasma shots required for a given experiment will be significantly reduced and problems with shotto-shot variations will be minimized. In addition, irreproducible events, such as the growth of plasma instabilities, can be studied. Impurity injection experiments to monitor the transport properties of the plasma, are also planned.

\section{III.C.5 References}

1. F. H. Coensgen, TMX Major Project Proposa1, LLL-Prop-148, January 12, 1977. 


\section{LABORATORY STUDIES}

(R. Bell, J. Castracane, M. Finkenthal, W. Hodge, W. Moos)

\section{$\underline{\text { Introduction }}$}

This section describes a series of studies performed in support of our instrumentation work and diagnostic studies. The first section describes the calibration procedures used in the absolute calibration of a grazing incidence monochromator. The second describes a series of extensive grating reflectivity measurements. The third describes a correlative study of tokamak and solar emissions.

IV.A. Calibration of Grazing Incidence Instrument with the SURF facility

As a result of the necessity to measure the absolute intensities line radiation in the $60-500 \AA$ range, a one meter grazing incidence monochromator was photometrically calibrated on the Synchrotron Ultraviolet Radiation Facility (SURF II) at the National Bureau of Standards. (The use of an absolutely calibrated monochromator as a diagnostic device on the Alcator $\mathrm{A}$ and $\mathrm{C}$ tokamaks allowed the measurement of total radiated power from high-Z impurity ions in the plasma.) A description of the instrumentation can be found elsewhere. ${ }^{1}$ The calibration procedure is described here briefly.

An absolute photometric calibration from 60 to $500 \AA$ was performed at SURF II using $243 \mathrm{MeV}$ electrons with between 2 - $10 \mathrm{~mA}$ of current in the storage ring. Since the radiation is highly polarized in the plane of the electron orbit and the grating is a partial polarizer, the calibration was performed in two orientations of the plane of dispersion: parallel and perpendicular to the plane of the electron orbit. Because light from a tokamak is not polarized, the results from the two orientations were 
àveraged together.

Synchrotron radiation is also highly collimated and will not fill the optics of a typical spectrometer. Therefore, it is necessary to map the grating in order to detect any variation in efficiency over the face of the grating. The spectrometer was rotated about the entrance slit in the plane of dispersion in order to illuminate different parts of the grating; no noticeable variation was found. A calibration run was performed by ocanning the wavelength while monitoring both the detector signal and the storage ring electron current. Calibrations were performed using the three filters in succession and finally with no filter on the optical axis. Fig. IV-1 displays the calibration curve for the $3.1 \times 10^{3} \AA$ thick C filter. The structure observed is due to silicon absorption edges from the glass in the channeltron. Fig. IV-2 is a graph of raw data displaying structure due to changes in efficiency of the channeltron near the $\mathrm{Si} \mathrm{L}$ absorption edges. The $44 \& \mathrm{C}$ absorption edge could not be observed with either C filter at an electron energy of $243 \mathrm{MeV}$, indicating that the intensity of radiation at the position corresponding to $44 \AA$ is largely composed of scattered light. The $\mathrm{C}$ absorption edge was barely visible through the thick C filter at $252 \mathrm{MeV}$. The thick carbon filter calibration will be used for the 60-200 $\AA$ range and the open position calibration for 200-500 . The beryllium $\mathrm{K}$ absorption edge at $111 \AA$ was obvious. The instrument is currently mounted on the Alcator C tokamak at the National Magnet Laboratory at MIT.

\section{$\underline{\text { References }}$}

1. W. L. Hodge, J. Castracane and H. W. Moos, Nuclear Instruments and Methods, to be published. 


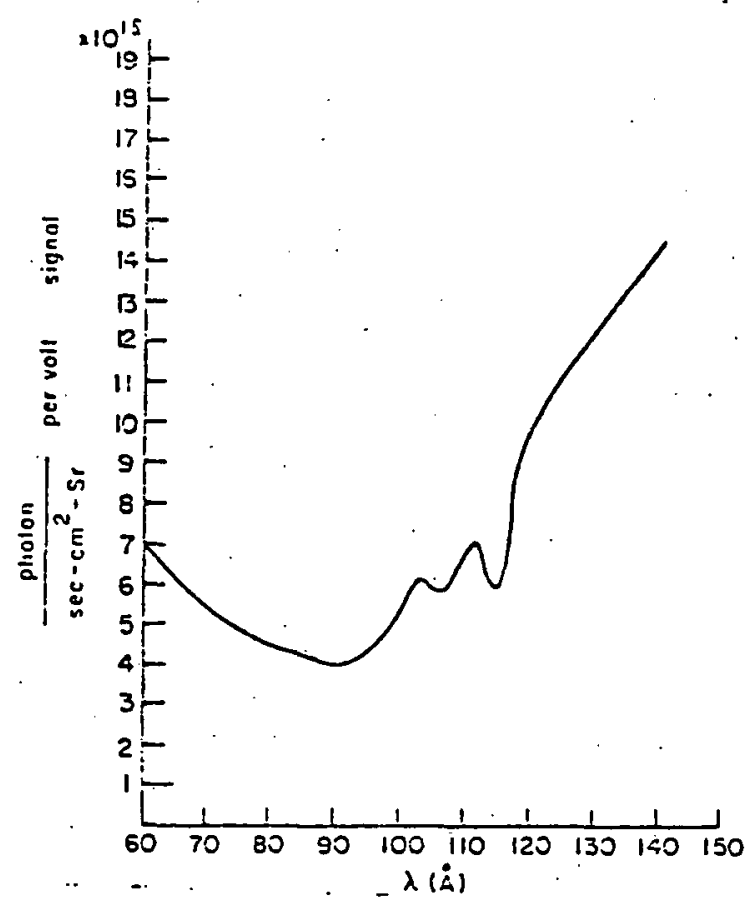

Fig. IV-1. Calibration curve for $3.1 \times 10^{3} \AA$ carbon filter.

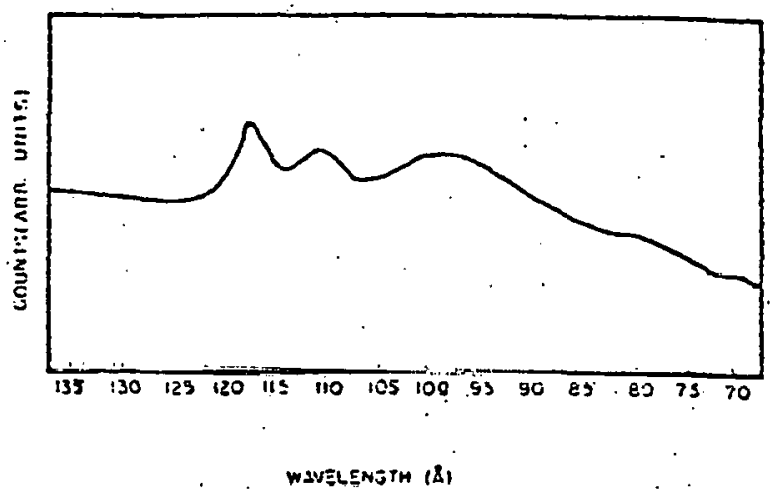

Fig. IV-2. Graph of typical calibration run $\left(3 \times 10^{3} \AA \mathrm{C}\right)$ showing structure due to silicon composition of channeleron. SURF was operating at $243 \mathrm{MeV}$ with approximately $4 \mathrm{~m} \mathrm{~A}$ of current. 


\section{IV.B Grating Efficiencies}

Four $400 \mathrm{~mm}$ gratings which were used in the Time-Resolving EUV. Spectrograph (see Section V) were evaluated over the spectral regions in which they would be used. Two instruments were used for this evaluation: a normal incidence one meter McPherson monochromator mounted with a comparator assembly and the Calibration and Test Equipment (CTE). The CTE which uses absolutely calibrated NBS photodiodes ạ transfor ctandards was used for wavelengths $\geq 1216 \AA$. The McPherson monochromator was used with a channeltron detector, and a good relative reflectivity measurement was made by comparing the light intensity illuminating the grating with that reflected from it. This system was used for wavelengths $\leq 1216 \AA$, and the absolute reflectivity was determined by normalizing the data at $1216 \AA$ to the measurements taken on the CTE. Two Bausch $\&$ Lomb gratings were evaluated: one $600 \mathrm{l} / \mathrm{m}$ gold coated grating blazed at $750 \AA$, Catalog Number 35-52-00-401; and one $12001 / \mathrm{mm} \mathrm{Al}$ and $\mathrm{MgF}_{2}$ coated grating blazed at $2064 \AA$, catalog number 35-52-00-720. Two Hyperfine gratings were also evaluated: a $36001 / \mathrm{mm}$ gold coated grating with a $300 \AA$ blaze; and a $24001 / \mathrm{mm} \mathrm{Al}$ and $\mathrm{MgF}_{2}$ grating with a $1000 \AA$ blaze. The reflectivities of the gratings appear in Figs. IV-3 to IV-6. 

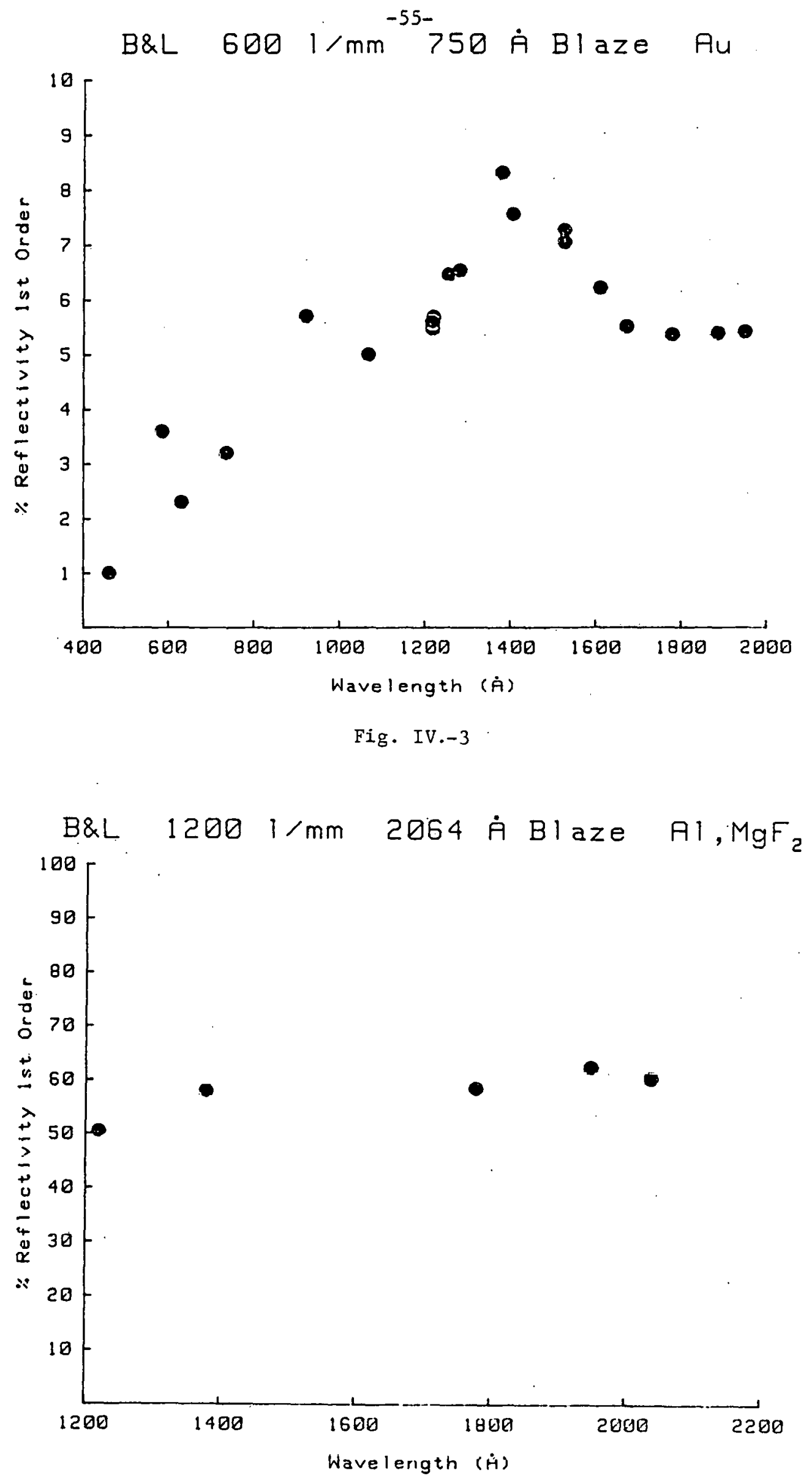

Fig. IV -4 
Hyperfine $36001 / \mathrm{mm} 300$ मे Blaze $\mathrm{Au}$

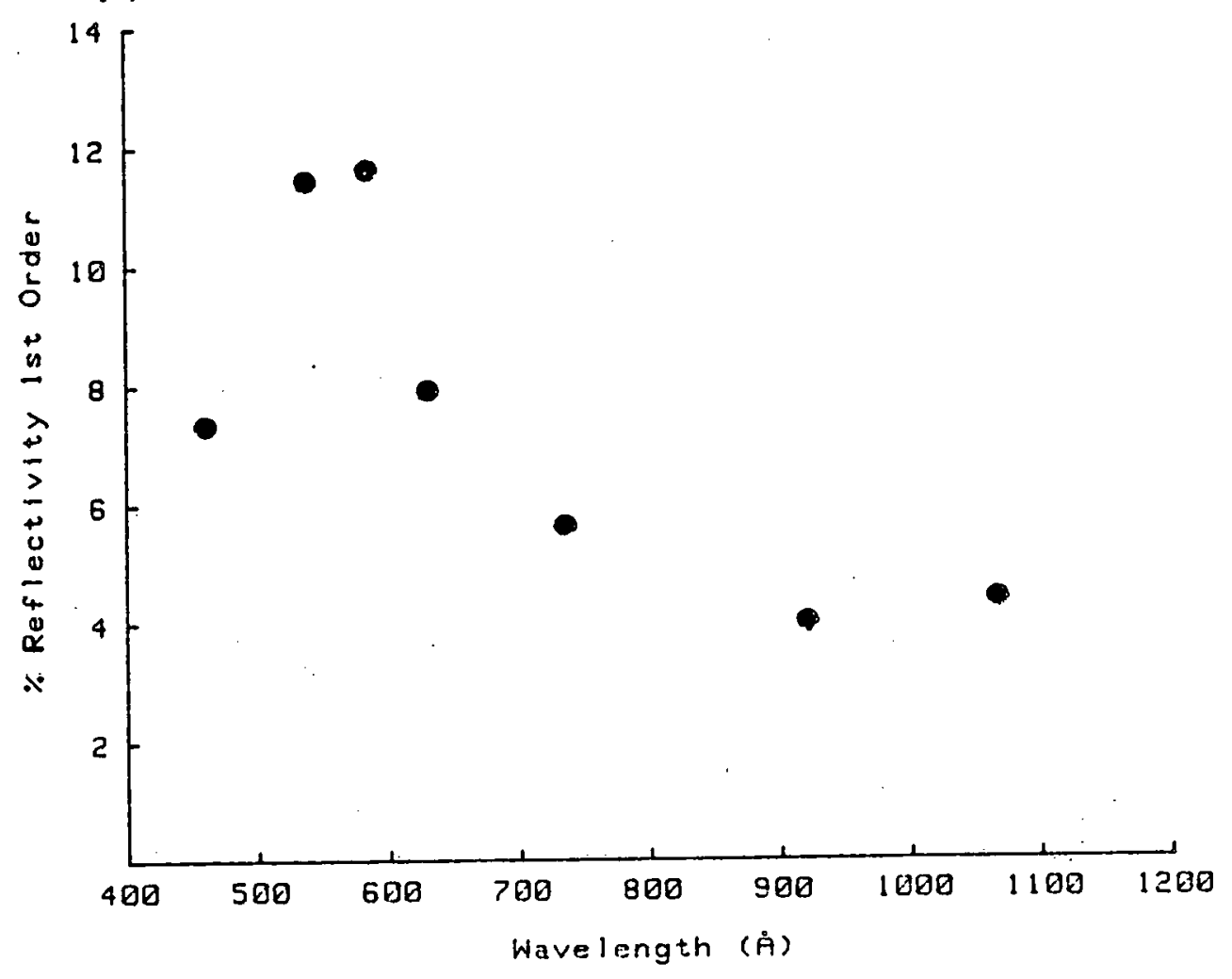

Fig. IV -5

Hyperfine $24001 / \mathrm{mm} 1000$ Aे Blaze Al, MgF

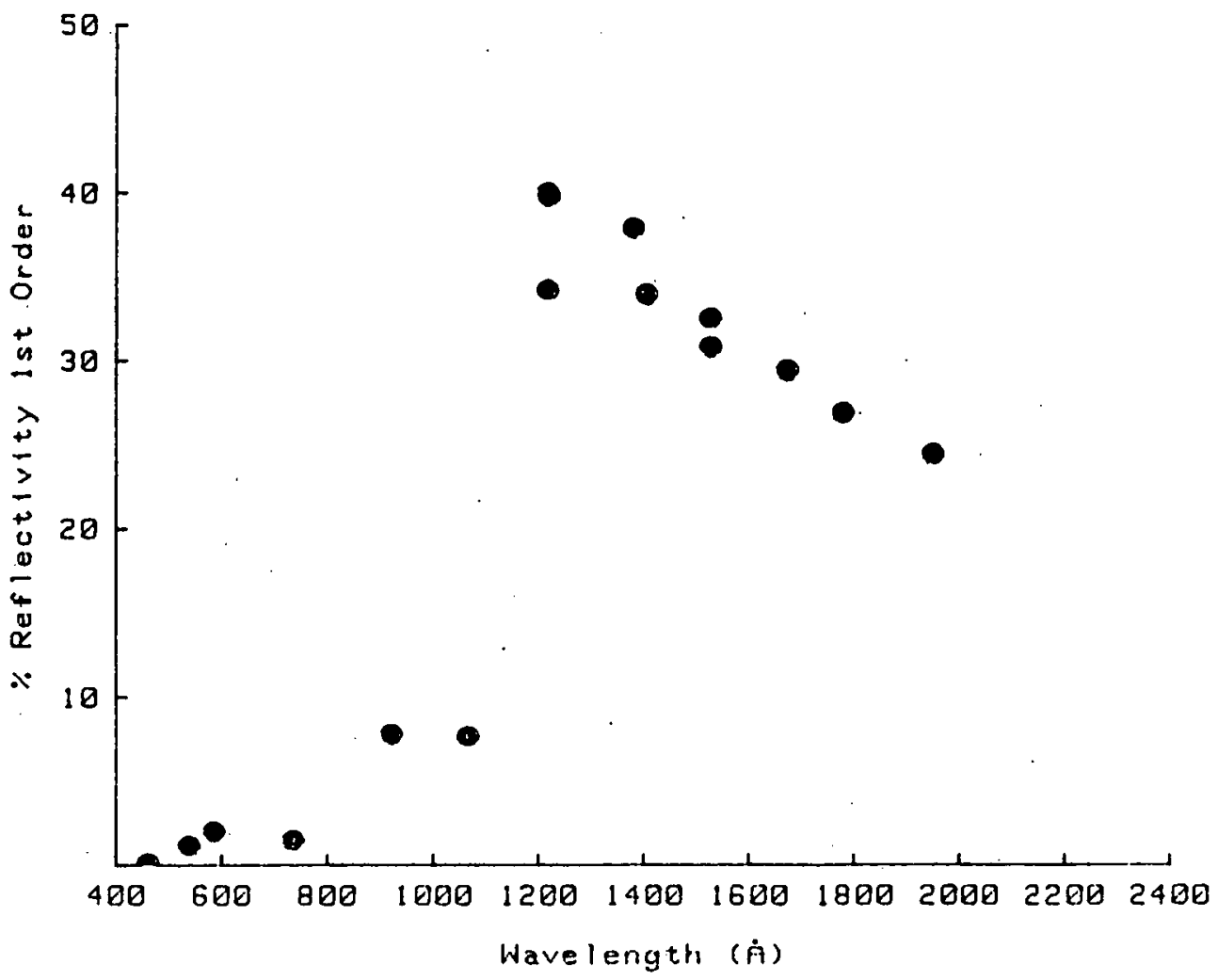

Fig. IV -6 
IV.C. Tokamak line study

This section describes work in progress on spectra obtained from the French TFR 600 tokamak. The study has three major goals:

1. To study the behavior of high $Z$ impurities in the 'cooler' part of a tokamak plasma $(100-500 \mathrm{eV})$.

2. To verify the accuracy of the atomic parameters used in the spectroscopy of high temperature plasmas.

3. To compare these spectra with those obtained during solar flares in order to improve the reliability of the verification of the atomic parameters.

The spectra have been recorded on photographic plates (Kodak SC5) in the 10 - $400 \AA$ range, using a high resolution one meter, grazing incidence spectrograph. A 600 groove/mm holographic J-Y grating was used to cover the entire range, while a $2400 \mathrm{~g} / \mathrm{mm}$ Bausch \& Lomb gold coat grating was used to obtain high resolution spectra in the $10-90 \AA$ range. A duo-chromator provided with photo-electric detection (two tungsten cathode magnetic multipliers) and the capability to scan the plasma radially was used complementarily in the $100-400 \AA$ region in order to check the line identifications in the photographic spectrum. : The main parameters of a typical TFR 600 discharge were: $T_{e}(0) \simeq 1=1.5 \mathrm{KeV}$ at $\mathrm{n}_{\mathrm{e}} \sim 10^{14} \mathrm{~cm}^{-3}$ or $\mathrm{T}_{\mathrm{e}}(0) \simeq 1.5-2 \mathrm{KeV}$ at $\mathrm{n}_{\mathrm{e}} \sim 3-5 \times 10^{13} \mathrm{~cm}^{-3}$ while the plasma current was $\sim 150 \mathrm{kA}$. The toroidal magnetic field $B_{t}=30-40 \mathrm{kG}$, and the discharge duration varied between $200^{\circ}-400 \mathrm{msec}$. Spectra were obtained both during ohmic \& $R F$ heated discharges. The small radius of the plasma varied between $17-21 \mathrm{~cm}$ and spectra were obtained at different radial positions and both during the current (and $\mathrm{T}_{e}$ ) rising and steady-state phase. 
The new version of the TFR tokamak, TFR 600 , had a new limiter (Inconnel 600) and new wall material (mostly stainless steel but also Inconnel 625). The spectra obtained were therefore used first to determine the nature of the impurities present in the discharge, their temporal behavior and how do they relate to the main plasma parameters - and finally how do they affect the power balance (by line radiation energy losses). The analysis gave the following results: the main metallic impurities have been found to be nickel $(z=28)$, chromium $(z=24)$, and iron $(z=26)$. The highest degrees of ionization observed are Li=like ions of those elements 1,2 Other impurities such as chlorine and manganese exist in the plasma and their behavior and spectra have been studied. $34^{4}$. An interesting correlation between the temporal behavior of highly ionized metallic species (ionization potential $>1 \mathrm{KeV}$ ) and the electron temperature profile has been noticed. 5 The overall amount of high $Z$ impurities is low in TFR- 600 plasma (Zeff $\simeq 1$ in most experiments) and the power losses by line radiation do not exceed those measured on TFR 400.5

Most of the above mentioned conclusions were drawn from the study of the highly ionized metallic species. As a general trend in tokamak spectroscopy the impurity behavior outside the hot core of the plasma (about $1 / 2<r / a<1$ ) is inferred from the study of line emission from low $\mathrm{Z}$ impurities (oxygen, nitrogen, carbon). But it is very probable that the way the impurities are introduced into the plasma will influence their further behavior ${ }^{5}$ and there is clear evidence that the release mechanisms depend primarily on the nature of the impurities and the nature of their link to the metallic structure. ${ }^{6}$ This is one important reason to study the behavior of lower stages of ionization of high $\mathrm{Z}$ elements and to compare them with low $\mathrm{Z}$ impurity ions with comparable ionization 
potentials. (For instance, is the evolution of $\mathrm{Cr}$ VI different from that of OV?). On the other hand, RF heating experiments on the Princeton PLT tokamak, seem to indicate that besides the net heating effect, the ICRF heating may reduce metallic radiation from the core through the increase of peripheral cooling by the light elements. The effect has not been observed on TFR 600, and a good way to check it further is to observe the lower stages of ionization of high $Z$ elements. But in order to study those states of ionization, the spectra must be analyzed and lines belonging to different ions, together with their relative (at least) intensities must be identified in the spectra. This work has been completed very recently. The line spectrum of chromium, manganese, iron and nickel in the isoelectronic sequences KI to NaI have been analyzed. Lines belonging to $3 s^{2} 3 p^{K}-3 s 3 p^{K+1}$ and $3 p^{K}-3 p^{K-1} 3 d$ transitions have been identified in the spectral range $100-300 \AA$. The ions studied cover the above mentioned radial range: Cr VI, IP $\sim 90 \mathrm{eV}-\mathrm{NiXVIII,} \mathrm{IP} \sim 600 \mathrm{eV}$.

During the steady-state phase of a tokamak discharge, a corona ionization equilibrium can be assumed (in some tokamaks, TFR among them, the assumption is confirmed by the comparison of experimental data and theoretical computations, see ref. 2 for the high $Z$ ions). At the relatively high densities at which they exist $\left(\mathrm{n}_{\mathrm{e}} \sim 10^{13} \mathrm{~cm}^{-3}\right)$, ions belonging to $\mathrm{KI}$ to NaI isoelectronic sequences have the populations of their ground levels at Boltzman equilibrium. ${ }^{7}$ In this case it can be shown that the line intensity ratios of different $\Delta \mathrm{n}=0$ transitions will depend on the collision strengths of the trañsitions under discussion. Therefore a comparison between experimental data and predicted intensity ratios will give valuable information on the atomic parameters used in computation. An analysis of the Fe VIII to Fe XV ions is in progress. 
As the ionization stage increases, higher densities are required in order to attain Boltzmann equilibrium in the ground state. ${ }^{7}$ Therefore the populations of excited states of many level ground state ions, will depend on the electron density. ${ }^{8}$ In this case, line intensity ratios will be a good density measure. Moreover using different elements ( $\mathrm{Cr}$ to Ni) lines ratios belonging to the same isoelectronic sequence will be density dependent over a large density range. A qualitative discussion of this topic has been done previously. 9 We are now comparing the tokamak spectra in the OI, NI and. CI isoelectronic sequence with solar flare spectra. The main problem is that a relative intensity calibration of the spectrum recorded on plates is difficult because of many blends. (There is already an overlap of lines belonging to different isoelectronic sequences of the same element in the case of $2 s^{2} s p^{K}-2 s 2 p^{K+1}$ transitions; in the tokamak spectra lines belonging to different elements are recorded in the same exposure.) If a satisfactory calibration can be achieved, this work will be completed in the very near future. 


\section{References}

1. C. Breton, C. De Michelis, M. Finkenthal, M. Mattioli, $2 s^{2} 2 p^{K}-2 s 2 p^{K+1}$ transitions in FI to BeI isoelectronic sequences of chromium, iron and nickel from tokamak produced plasmas. J. Opt. Soc. Am. 69, 12, (1979).

2. TFR Group, Are tokamak plasmas at corona equilibrium?, Rep. EUR-CEA, February 1980.

3. TFR Group, Chlorine VUV line emission from TFR 600 tokamak plasmas, Rep. EUR CEA FC 984, January 1979.

4. TFR Group, The spectrum of Mn XVII (FI-like) Fo Mn XXII (BeI) like ions emitted by a tokamak produced plasma, Phys. Lett 74A, 1979.

5. C. Breton, D. De Michelis, M. Finkenthal, M. Mattioli, Space and Time resolved impurity studies on TFR 600 plasmas, DPh-PFC NT 229 (Internal Report), December 1979.

6. G. M. McCracken, P. E. Stott, Plasma-Surface interactions in tokamaks, (Review Paper), Nuclear Fusion 19, 7, 1979.

7. K. P. Dere, H. E. Mason, K. G. Winding, and A. K. Bhatia, XUV electron density diagnostics for solar flares, Ap. J. 40, 341, 1979.

8. U. Feldman and G. A. Doschek, Plasma diagnostics using high-resolution spectroscopic techniques, J. 0pt. Soc. Am. 67, 6, 1977.

9. C. Breton, C. De Michelis, M. Finkenthal, M. Mattioli, The relevance of tokamak plasmas for astrophysics, paper presented at the SAS Symposium, Tucson, Arizona, September 1979. 


\section{INSTRUMENTATION}

(R. Bell, R. Dukart, W. Hodge, R. Holman, M. Finkenthal, B. Stratton)

\section{V.A. Time-Resolving EUV Spectrograph}

In the previous progress report, a 1024 element detector with spectrographic capabilities was described. In the past year, such a detector and a suitable spectrograph have been designed and constructed. The spectrograph will provide time-resolved measurements over a wide spectral range in a single plasma shot $(300-2200 \AA)$. This is a very important improvement in the spectroscopic diagnostics of a tokamak plasma. It will enable us to study simultaneously the behavior of a large number of ionization states belonging to a given impurity, covering both the cooler part and the hot core of the plasma. Moreover it will record simultaneously the spectrum of both light and heavy impurities radiating at different radii. All this will allow a better understanding and a more rapid interpretation of the interplay between the light and heavy impurities during the discharge. The fact that such a large spectral range is covered will permit the study of the physical background versus line radiation in the EUV region. Forbidden lines in EUV have been used in order to measure the ion temperature, but still a thorough study of their electron density dependence has to be done. This instrument is not only best suited for this study, but for certain elements both resonant and forbidden lines will fall within its spectral range. It may also serve as a survey instrument during, for instance, heating experiments, or whenever a rapid evaluation of a radical change in one of the main plasma parameters (such as $\mathrm{T}_{e}, \mathrm{~N}_{e}, \mathrm{I}$, etc.) is needed.

The detector is a windowless image intensified Reticon photodiode array, 
(see Fig. V-1.) with a CuI photocathode. The photocathode was deposited directly on a $40 \mathrm{~mm}$ microchannel plate (MCP). Behind the MCP is an aluminized phosphor screen and a fiber optic bundle to which the 1024 element photodiode array is attached. Each photosensitive element (pixel) on the Reticon array is 25 microns wide and $2.5 \mathrm{~mm}$ high. Reducing fiber optics are used to map a $4 \times 40 \mathrm{~mm}$ area of the MCP onto the $2.5 \times 25.6 \mathrm{~mm}$ photosensitive area of the photodiode array. The body of the image tube is welded to a $3-3 / 8$ inch conflat flange.

The detector works as shown in Fig. V-2. When an EUV photon strikes the photocathode, the resulting photoelectron undergoes channel multiplication in the MCP. A variable potential of between 400 volts and 900 volts controls the electron gain in the multiplication. A shower of electrons exit the back of the MCP and are proximity focused by a 5000 volt potential into a P-20 phosphor. Visible photons created in the phosphor are then channeled through fiber optics to a Reticon photodiode array. The photocurrent from the visible photons depletes the charge on the photodiodes. After some exposure time, scanning circuitry will recharge each photodiode in sequence, and a voltage train of pulses, each proportional to the light incident on a particular dinde, will be output. This voltage train can then be digitized and stored in a computer to be processed.

In order to choose an optimum thickness for the CuI photocathode, several thicknesses of CuI were deposited on MCP's and the quantum efficiencies were measured. The MCP's were used as a substrate that would be similar to what was used in the final detector. The quantum efficiency was measured by illuminating a $5 \mathrm{~mm}$ spot of the CuI and measuring the resulting photoelectrons with a channeltron. These measurements were carried out for wavelengths between $460 \AA$ and $2500 \AA$. 


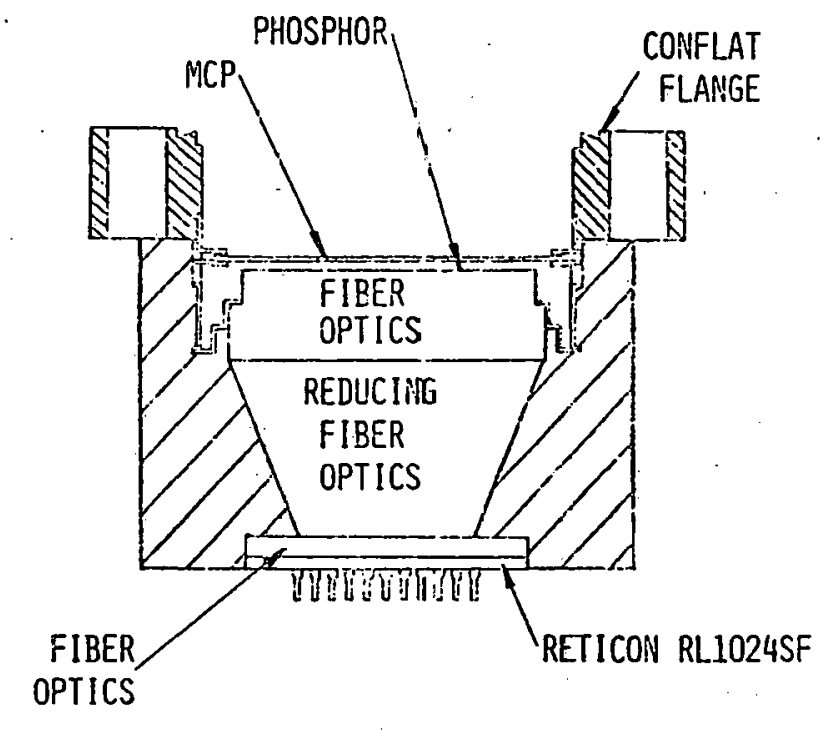

WINDONLESS IMAGE INTENSIFIED PHOTODIODE ARRAY

Fig. V-1

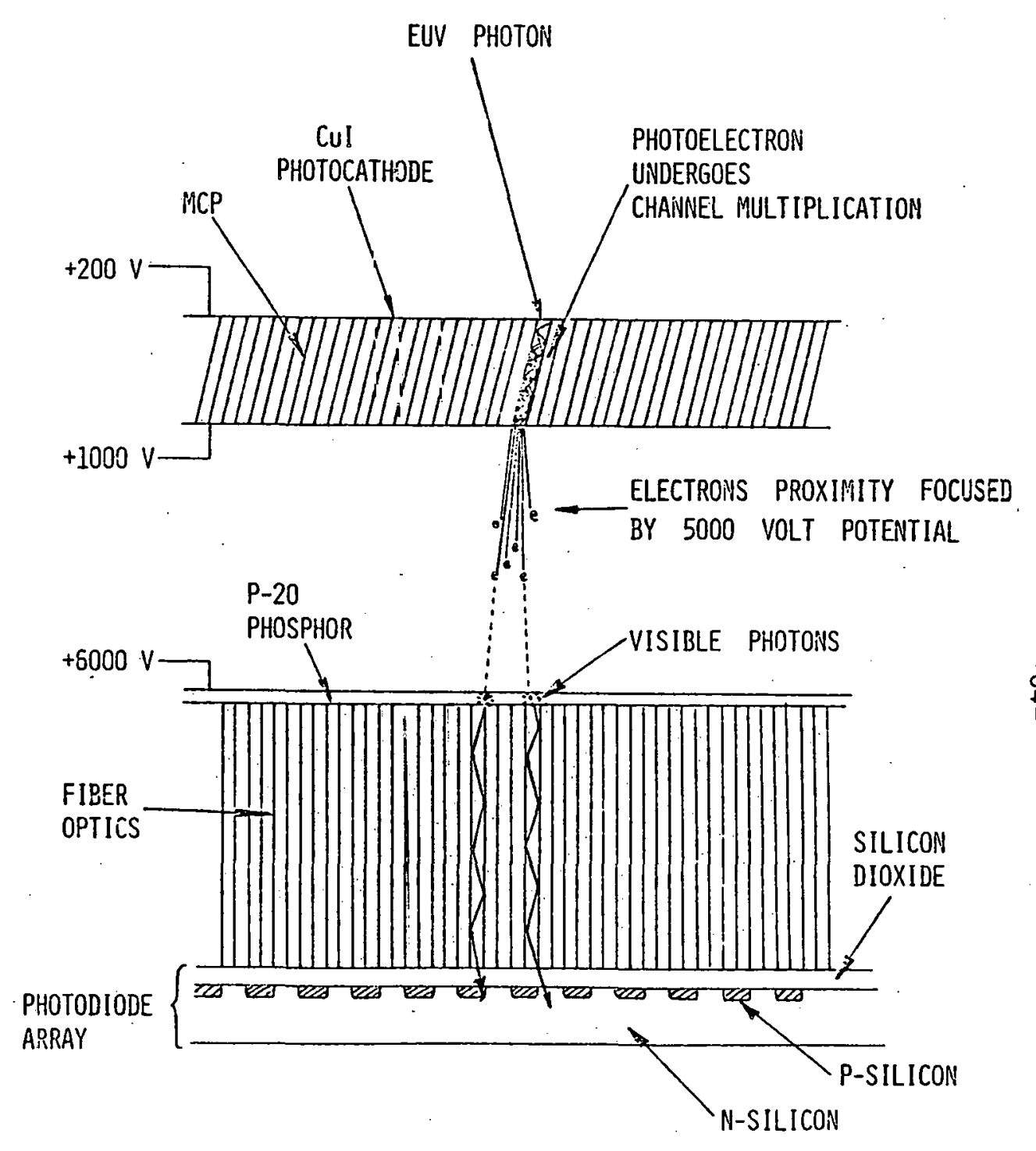

吕 
It was seen that the quantum efficiency of the CuI exceeded that of a bare MCP over this entire region. Unexpectedly the CuI had a useable quantum efficiency at wavelengths up to $2200 \AA$, and as a result attempts were made to extend the range of the spectrograph to $2200 \AA$. The CuI also seems to lose some of its quantum efficiency when exposed to air for some time, perhaps due to the absorption of water vapor. However, the CuI would recover its quantum efficiency if it was left in a vacuum for several days. The quantum efficiency, for example, would increase by as much as $100 \%$ between one day and three days in vacuum. A CuI thickness of $\sim 1140 \AA$ was chosen for the detector.

The windowless image intensifier is a modification of the standard F-4111 image intensifier tube from the ITT Tube and Sensor Laboratories. The modification eliminates the front window and transmission photocathode and includes mounting on a conflat flange and direct deposition of the CuI photocathode on the MCP. A standard F-4111 image intensifier was used to determine the resolution of the detector. A ten to fifteen micron spot was imaged on the front of the image intensifier and the intensified image appearing at the fiber optic output was measured with a scanning photomultiplier tube. The full width half maximum of the image was measured to be $50 \mu \mathrm{m}$.

The detector was evaluated on the Extreme Ultraviolet Calibration Equipment (ECE) to determine.its quantum efficiency, gain and linearity. The quantum efficiency times gain (QG) versus wavelength is shown in Fig. $\quad-4$. The gain of the tube can be estimated using the quantum efficiency measurement of CuI. The approximate electron gain per photoevent (i.e. electrons produced in the Reticon per photoevent) is 
Quantum Efficiency of CuI Photocathode

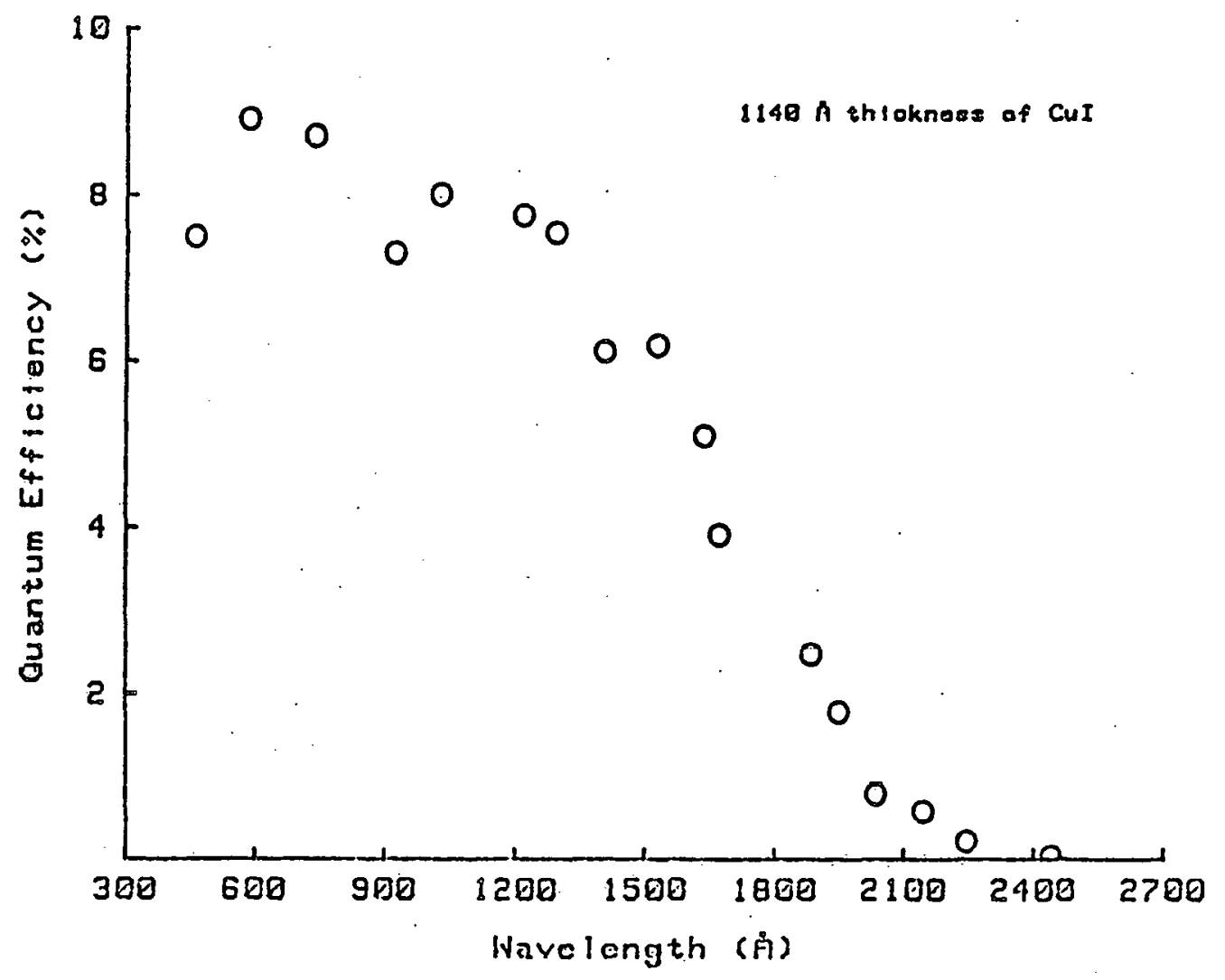

Fig. $V-3$

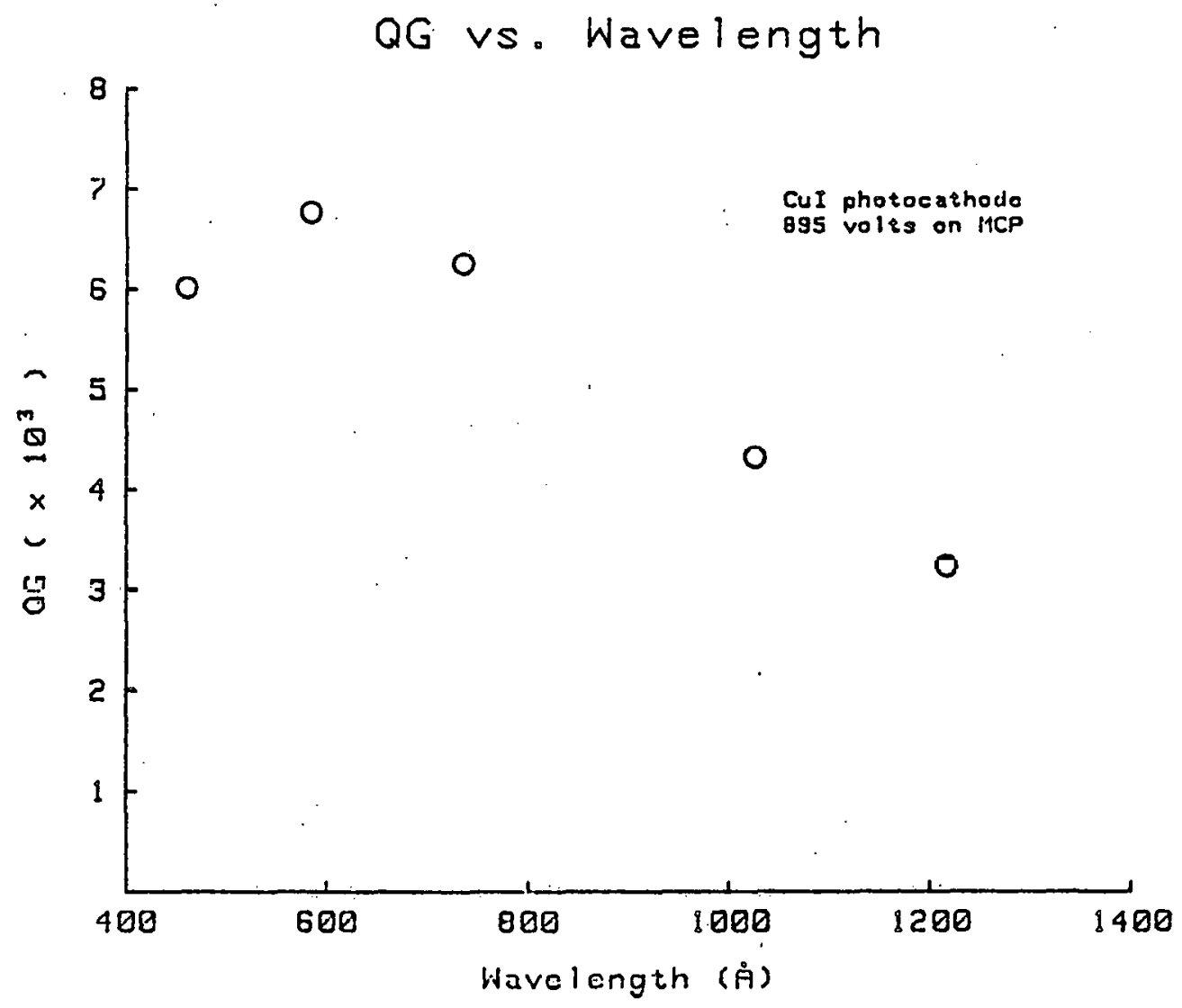

Fig. $V-4$ 
$4 \times 10^{4}$ at a MCP voltage of 895 volts. The gain of the detector can be varied over about three orders of magnitude (see Fig. V-5).

The response of the detector remained linear up to the maximum flux density that the ECE could achieve (see, Fig. V-6). The peak signal on Fig. V-6 corresponds to $110-15 \%$ of the MCP strip current density.

The spectrograph is a $400 \mathrm{~mm} \mathrm{f} / 30$ normal incidence system w1th seven gratings, each of which is fixed with respect to the entrance slit and detector. The gratings are $13 \mathrm{~mm} \times 13 \mathrm{~mm}$ in size and are mounted in two rows. Each row is slightly above or below the plane of the Rowland Circle. Two of the gratings have relatively low dispersion and cover the entire spectral range between $300 \AA$ and $2180 \AA$. The other five gratings have higher dispersion and cover adjacent intervals of the spectrum. (See Table V-1). A preslit in front of the entrance slit insures that only one grating at a time is illuminated. The instrument is rotated about the entrance slit to bring a particular grating in line with the beam defined by the preslit and entrance slit.

\section{GRATINGS}

\begin{tabular}{lcccc}
$1 / \mathrm{mm}$ & Blaze $(\AA)$ & Overcoating & Range $(\AA)$ & Resolution $(\AA)$ \\
\hline 600 & 750 & $\mathrm{Au}$ & $250-1910$ & $\sim 4.1$ \\
3600 & 300 & $\mathrm{Au}$ & $300-578$ & $\sim .7$ \\
3600 & 300 & $\mathrm{Au}$ & $550-828$ & $\sim .7$ \\
3600 & 300 & $\mathrm{Au}$ & $800-1078$ & $\sim .7$ \\
2400 & 1000 & $\mathrm{Al}, \mathrm{MgF} 2$ & $1051-1467$ & $\sim 1.0$ \\
2400 & 1000 & $\mathrm{Al}, \mathrm{MgF}$ & $1451-1867$ & $\sim 1.0$ \\
1200 & 2064 & $\mathrm{Al}, \mathrm{MgF}_{2}$ & $1349-2183$ & $\sim 2.1$
\end{tabular}




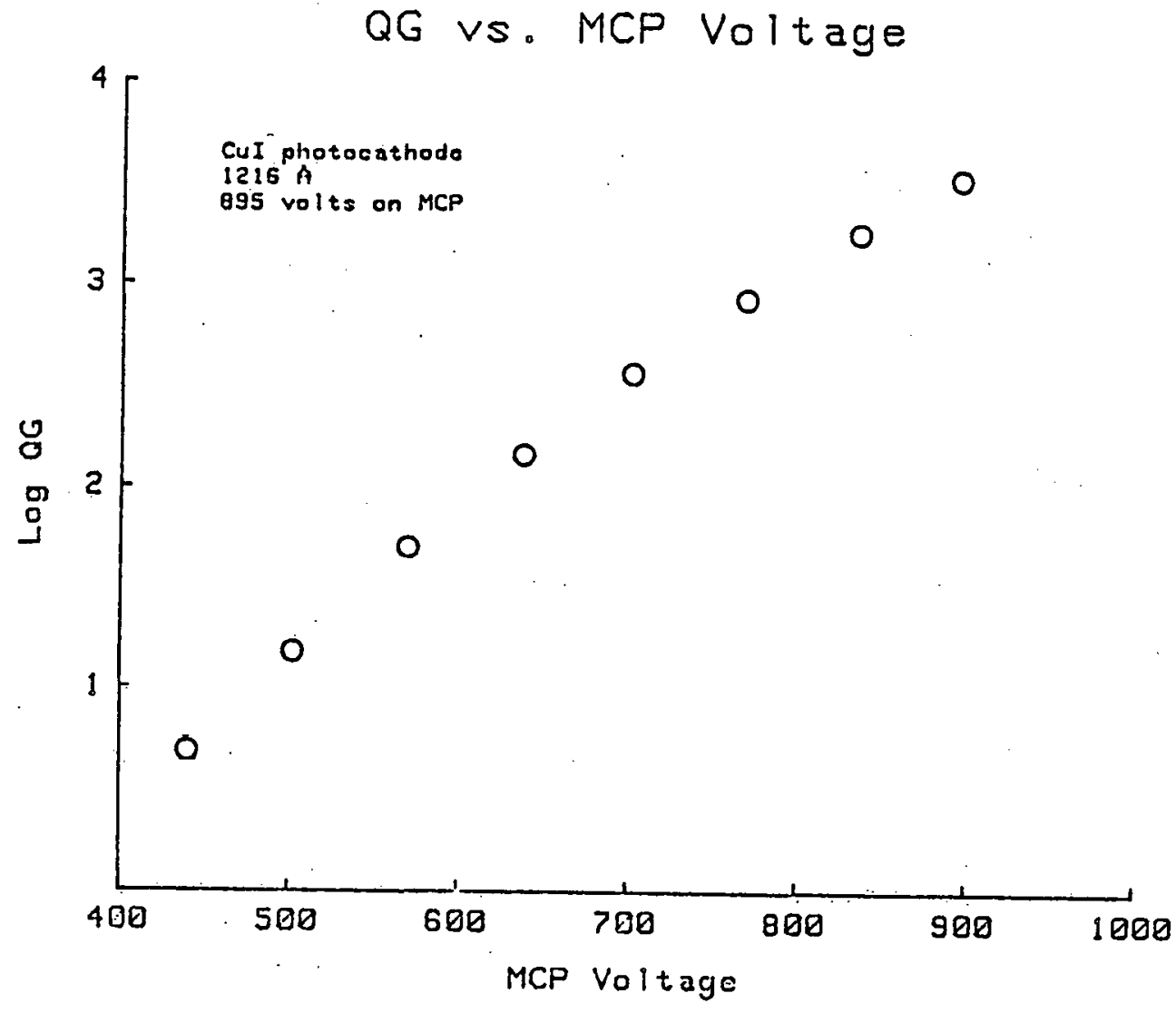

Fig. V -5

Signal vs. Flux Density

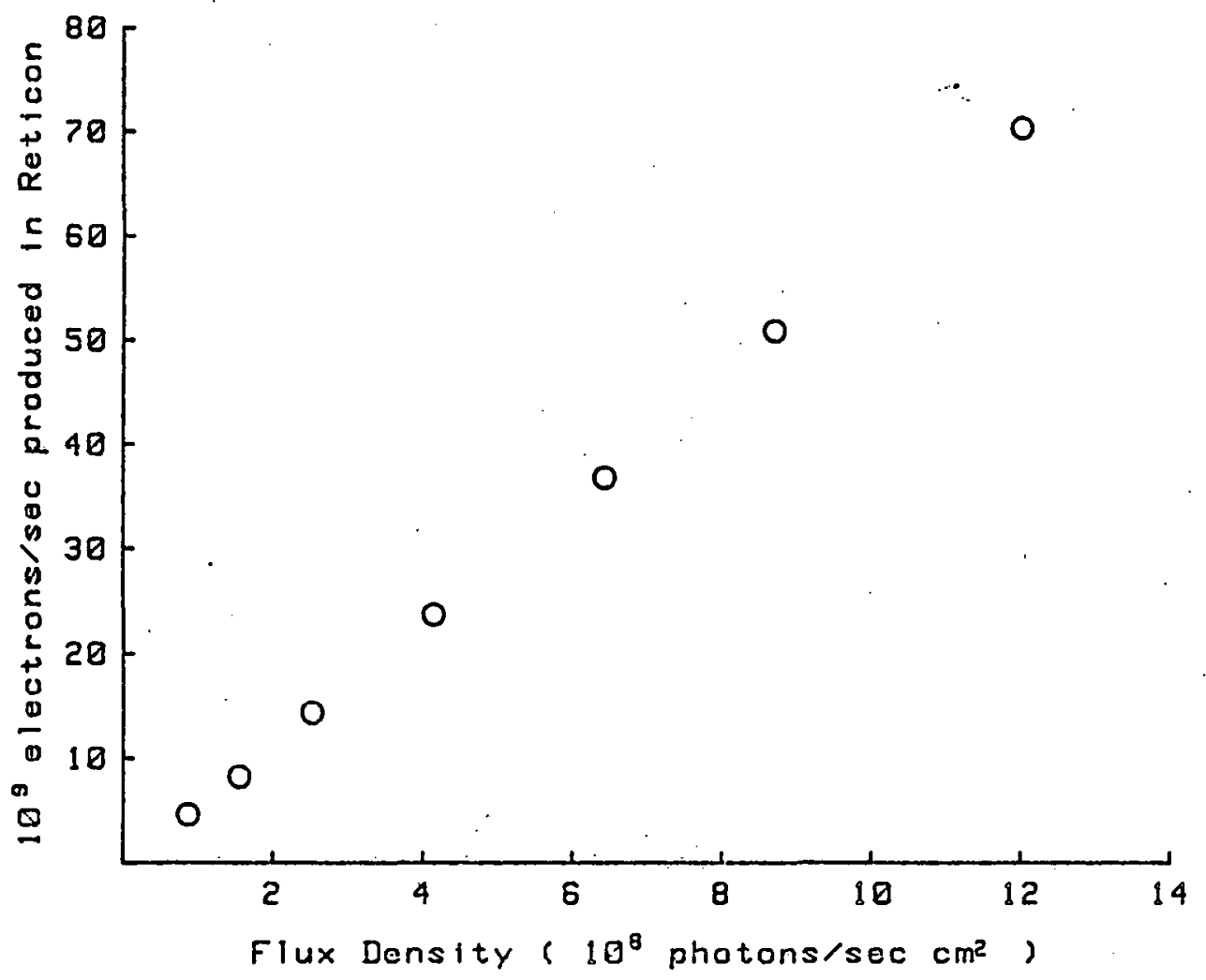

Fig. V-6 
This system of several fixed gratings takes advantage of the properties of an extended detector. A variable wavelength drive is not needed since a range of wavelengths can be seen by the detector simultaneously. Only discrete positions of the grating along the Rowland circle are required to cover a wide spectral range. A grating fixed with respect to the entrance slit and detector means that the wavelength that falls on a particular pixel is fixed. This makes both wavelength and photometric calibration easier since a single pixel is calibrated for a single wavelength. This also eliminates the need for a high precision drive to move the grating along the Rowland circle, and the drive mechanism that is required to rotate the instrument is outside the vacuum where standard mechanical techniques (such as using oil for lubrication) can be applied. Using several gratings allows the blaze, the overcoating and the dispersion to be chosen independently for each grating. An overcoating of gold, for example, can be used below $1200 \AA$, and an overcoating of aluminum and magnesium fluoride can be used above $1200 \AA$ to enhance the reflectivity of the grating. The blaze of each grating can also be chosen to peak in the wavelength region covered by a particular grating. When only a single grating is used a compromise must be made between wide spectral coverages and high resolution. If both a low dispersion and high dispersion grating are used, no compromise is necessary.

The data acquisition system is shown in Fig. V-8. A Reticon scanning circuit recharges the Reticon photodiode array and outputs a train of voltage pulses which are digitized by an A/D to 12 bit resolution. The digitized signal plus timing information are placed in a buffer which is read into an HP 9835 Desktop computer by Direct Memory Access (DMA). 


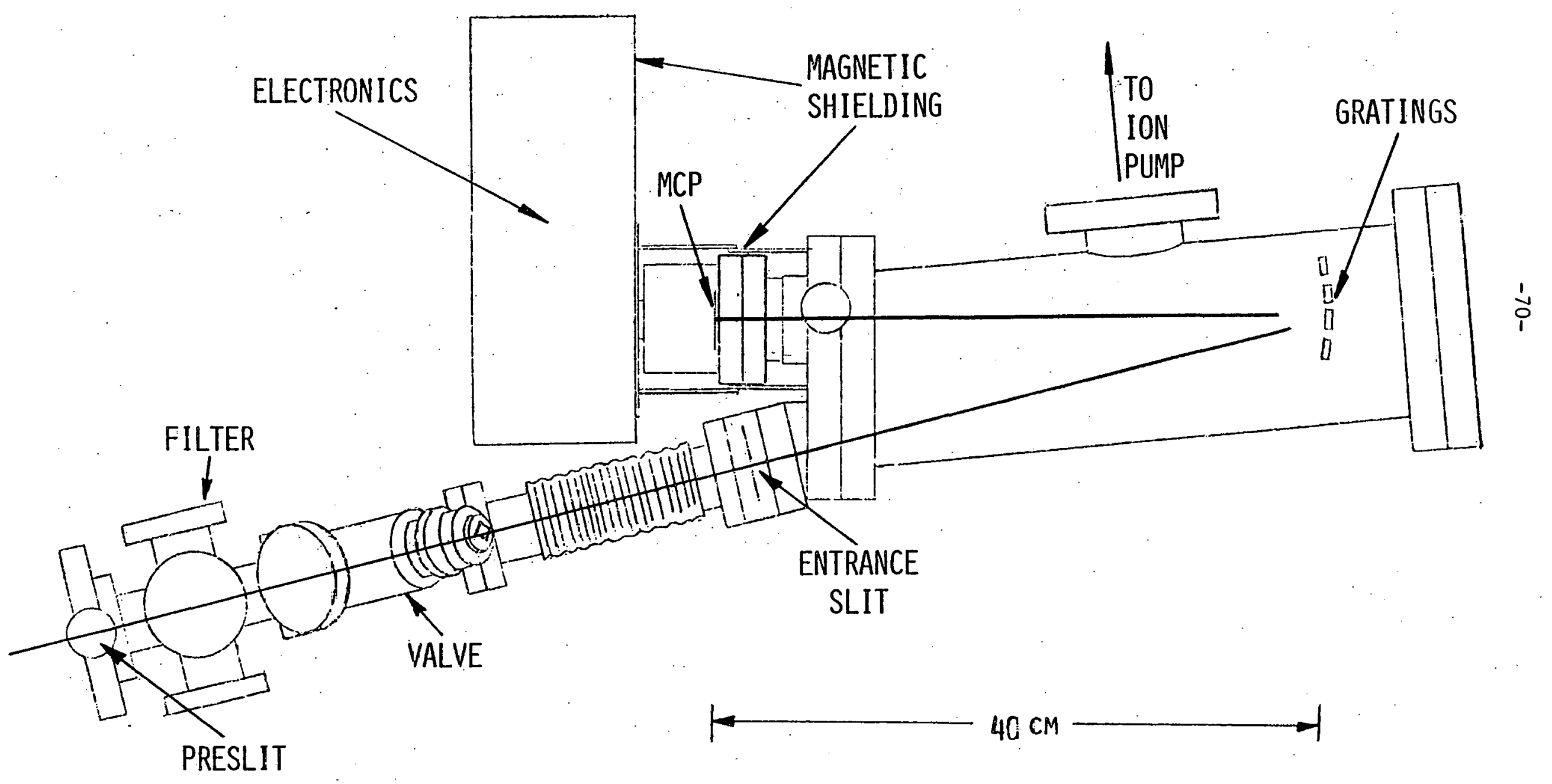

Fig. V-7 


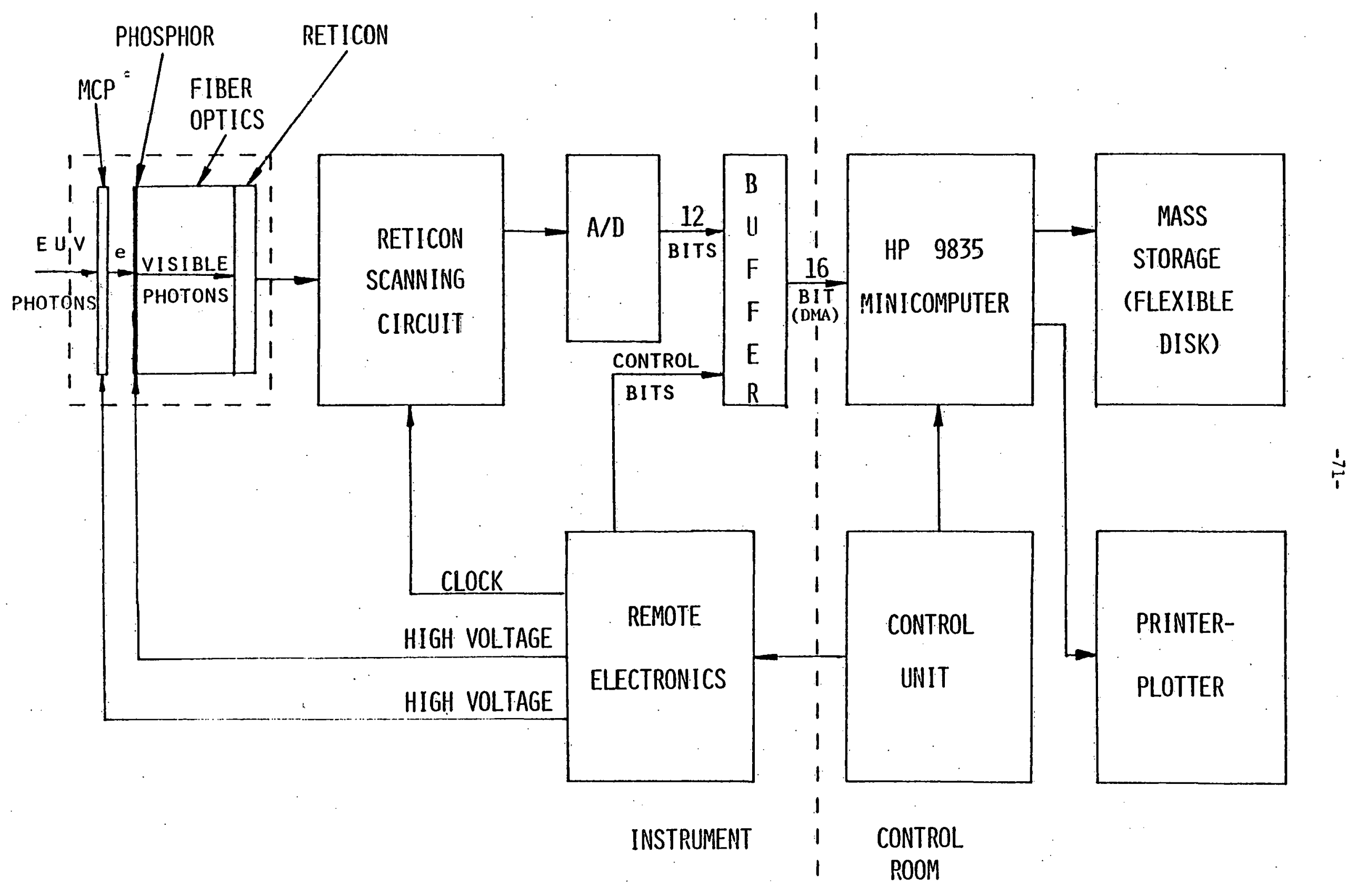

Fig. V-8 
The DMA rate of the computer is about equal to the maximum rate of operation of the scanning circuitry; 280 kHz. An entire spectrum of 1024 pixels can be read into the computer every $4 \mathrm{~ms}$. The computer has a large memory capacity and can record the spectrum for up to 100 time periods in one plasma shot. The computer then processes the data, such as folding in the calibration, etc. and outputs the information graphically between plasma shots. Data is stored on flexible disk. 


\section{V.B. Two Meter Grazing Incidence Double Monochromator}

An evaluation of the two meter grazing incidence double monochromator, described in previous annual reports, was undertaken during the past year. A pumping system, spectrometer stand, light source, and a single channel channeltron detector were assembled. A precise method of positioning the gratings on their support arms and of aligning these arms inside the spectrometer body during grating installation was developed. The spectrometer was evaluated as a single monochromator using a $133.61 / \mathrm{mm}$ grating, which gave a wavelength coverage region from $200 \AA$ to $600 \AA$. The resolution was determined to be within a factor of 2 of the theoretical resolution, including the effects of astigmatism. 


\section{V.C. Time-Resolving Grazing Incidence Spectrograph}

A time resolving one-meter grazing incidence spectrograph has been designed and is currently under construction. The instrument will be used to study the physics of high $\mathrm{Z}$ impurity ions in Tokamak plasmas. The grazing incidence region (soft X-ray 10-200 $\AA$ ) is in a spectral region in which heavy ions will radiate strongly, especially when they are in the central region of the plasma. The instrument will have a 50-100 \& spectral range and therefore will be able to study different transitions simultaneously from the same ionization state. Since the electron density and temperature are known within $15 \%$ on a tokamak, then the ratio of brightnesses of transition from the same ionization state can be used to predict atomic rate coefficients. These coefficients can then be compared to theoretical coefficients, giving an extremely powerful method to test theory with experiment.

Minuteman Laboratories, Acton, Massachusetts will supply the grazing incidence spectrograph; ITT Electro-Optics Division, Fort Wayne, Indiana will supply the multispectral detector system. The grazing incidence system will have a one meter Roland Circle diameter with a $12001 / \mathrm{mm}$ JY holographic grating and 10 micron entrance slits. All metal vacuum seals will keep high vacuum integrity and an oil free Varion Vac-ion pump will eliminate hydrocarbon contamination.

The detector'system is similar to the intensified Reticon system described in section V.A, thus only the major differences will be described. The major difference is in the fabrication of the microchannel plate. The photocathode will be $\mathrm{MgF}_{2}$ instead of $\mathrm{CuI}$. Because in a grazing incidence mount the radiation strikes the MCP at a high angle of incidence ( $20^{\circ}$ from the normal to the plate), a standard MCP would have a very low quantum 
efficiency. This problem is solved by funneling the plate. This process opens the area of the channels on the photocathode side of the plate, allowing the radiation to strike the plate closer to normal incidence and thus increasing the quantum efficiency.

The Space Sciences Lab, Berkeley, California has compared the quantum efficiencies of funneled and non-funneled MCP and found large increases (a factor of 30 at $170 \AA$ for a $80^{\circ}$ incidence angle) in the quantum efficiency of the funneled plates relative to the standard plates at large angles of incidence.

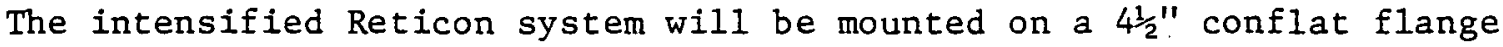
which will be bolted onto a detector housing. The detector housing will be moved along the Rowland circle so that the MCP will remain tangent to the Rowland circle. The reticon output will be digitized at the 8 bit level and then stored by direct memory access in a small computer. With a $12001 / \mathrm{mm}$ grating, total wavelength coverage will be from $10-350 \AA$ (.1 to .2 \& resolution). The spectral range over which simultaneous measurements can be made will be $20 \AA$ at $16 \AA, 50 \AA$ at $80 \AA$ and $75 \AA$ at $200 \AA$.

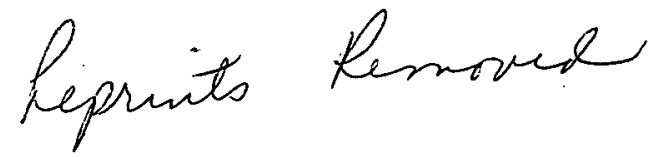


VI. PUBLICATIONS AND ABSTRACTS

W. Fielder, D. L. Lin and D. Ton-That, E1 and M2 Transitions in the Neon Isoelectric Sequence, Phys. Rev. A 19, 741 (1979).

N. H. Lazar, K. H. Carpenter, J. M. Tyson, E. S. Warden and H. W. Moos, Spatial Distribution of Impurities and $\mathrm{H}$ Neutrals in Elmo Bumpy Torus (EBT), Nuclear Fusion, 19, 571 (1979).

R. Paul Drake and H. Warren Moos, Evidence. for Neutral Beam Injected Oxygen Impurities in 2XIIB, Nuclear Fusion Letters 19, 407, (1979).

H. W. Moos, K. I. Chen, I. I. Terry and W. G. Fastie, Construction, Calibration and Application of a Compact Spectrophotometer for EUV (300-2500 §) Plasma Diagnostics, Appi. Opt。18, 1209 (1979).

E. S. Marmar, D. Overskei, H. Helava, K. I. Chen, J. L. Terry and H. W. Moos, The Effects of Wall Temperature on Light Impurities in Alcator, Nuclear Fusion Letters 19, 485 (1979).

L. Armstrong, Jr. and W. Fielder, Jr. Photoionization Cross-section using multi-configuration Hartree-Fock and its extension, Physica Scripta 21, 457, 1980.

W. R. Fielder, Jr. and Lloyd Armstrong, Jr., Interchannel Effects on $\mathrm{Cl}$ Photoionization Cross Sections, Bul. APS, 24, 1201 (1979).

R. K. Richards, H. W. Moos, and S. L. Allen, A Spatial Imaging Detector System for Pulsed Plasma Extreme U1traviolet Diagnostics, Review of Scientific Instruments, 51, 1 (1980).

W. Hodge, J. Castracane, W. Moos, Photometric Calibration of a One Meter Grazing Incidence Spectrometer for Plasma Fusion Diagnostics, to be published Nuclear Instruments \& Methods.

R. P. Drake and H. W. Moos, Extreme Ultraviolet Study of the 2 XIIB Neutra1-Beam-Heated Mirror Machine, to be published in Nuclear Fusion.

K. I. Chen, J. L. Terry, and H. W. Moos, Spatial Profiles of Light Impurity Ions in the Alcator A Tokamak Plasma, Nucl. Fusion 20, 189 (1980).

S. L, Allen, H. W. Moos, R. K. Richards, J, T. Terry, and E. S. Marmar, The Influence of the Limiter on EUV Emissions from Light Impurities in the Alcator A Tokamak, submitted to Nuclear Fusion.

E. S. Marmar, J. E. Rice and S. L. Allen, Confinement of Injected Silicon in the Alcator A Tokamak, submitted to Phys. Rev. Lett. 
H. W. Moos, Extreme Ultraviolet Diagnostics of High Temperature Plasmas, invited paper for U.S. - Japan Seminar on Plasma Spectroscopy, Kyoto, Japan, 7-11 May 1979.

R. P. Drake and H. W. Moos, Neon Injection Experiments in 2XIIB, 1979 IEEE International Conference on Plasma Science, June 4-6, 1979, Montreal.

H. W. Moos, S. L. Allen, R. E. Bell, and R. K. Richards, EUV Spatial Imaging and Multispectral Element Detector Systems, paper given at Department of Energy Workshop, Germantown, 31 May 1979.

R. E. Bell, H. W. Moos, A Multispectral Detector for an EUV Time-Resolving Spectrograph, Bul: APS, 24, 995 (1979).

O. T. Strand, H. W. Moos, R. S. Hornady, EUV Study of Impurities on TMX, $\mathrm{Bul}$. APS, 24, 1019 (1979).

B. Stratton, S. L. Allen, J. L. Terry, E. S. Marmar, Far Ultraviolet Emissions from the Alcator C Tokamak, Bul. APS, 24, 997 (1979).

S. L. Allen, E. S. Marmar, J. E. Rice, H. W. Moos, Diffusion and Confinement of Injected Impurities in the Alcator A Tokamak, Bul. APS, 24, 926 (1979).

W. Hodge, J. Castracane, H. W. Moos, E. S. Marmar, M. Pickrell, Photometrically Calibrated Spatially and Temporily Resolved Measurements of Mo Radiation in the Soft X-Ray Region on Alcator A, Bul. APS, 24, 926 (1979).

J. Castracane, W. Hodge, H. W. Moos, Photometrically Calibrated Study of the Soft X-Ray Region of Molybdenum in Alcator A, Third Workshop on Use of XUV and X-ray Radiometry in Plasma Diagnostics $5 \mathrm{eV}$ to $10 \mathrm{keV}$, NBS, Nov..19, 1979.

R. E. Bell and H. W. Moos, An EUV Time-Resolving Spectrograph for Plasma Diagnostics, Third Workshop on Use of XUV and X-ray Radiometry in Plasma Diagnostics $5 \mathrm{eV}$ to $10 \mathrm{keV}$, NBS, Nov. 19, 1979.

O. T. Strand, H. W. Moos, and R. S. Hornady, EUV Study of Impurities on TMS, paper given at Third APS Topical Conference on High Temperature Plasma Diagnostics, UCLA, March 17-19, 1980.

R. E. Bell, H. W. Moos, An EUV Time-Resolving Spectrograph, paper given at Third APS Topical Conference on High Temperature Plasma Diagnostics, UCLA, March 17-19, 1980.

W. Hodge, M. Finkiththa1, R. Bell, and H. W. Moos, Grazing Incidence and Norinal Incidence Spectrographs for Plasma Fusion Diagnostics, given at IEA Workshop on Tokamak Diagnostics, PPPC, Princeton, N.J. March 11-14, 1980.

S. L. Allen, H. W. Moos, E. S. Marmar, and J. E. Rice, A Study of Injected Impurities on the Alcator A Tokamak, IEEE International Conference on. Plasma Science, Univ. of Wisconsin, May 19-21, 1980. 


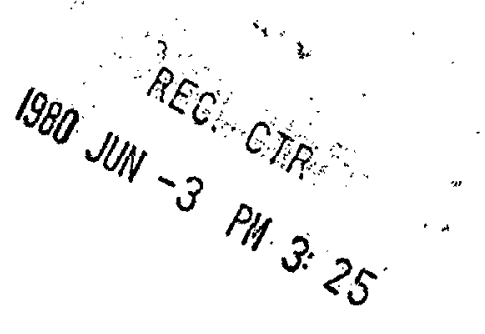

